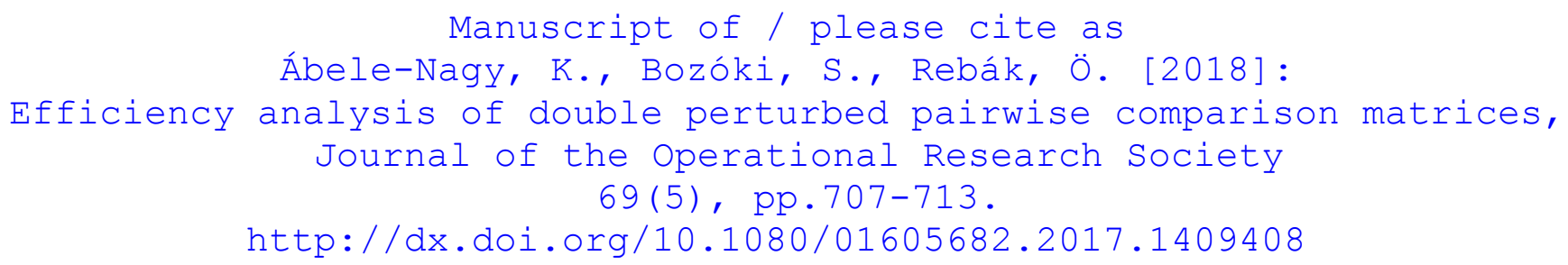

\title{
Efficiency analysis of double perturbed pairwise comparison matrices
}

\author{
Kristóf Ábele-Nagy ${ }^{1,2 *} \quad$ Sándor Bozóki ${ }^{1,2 \dagger} \quad$ Örs Rebák
}

\begin{abstract}
Efficiency is a core concept of multi-objective optimization problems and multi-attribute decision making. In the case of pairwise comparison matrices a weight vector is called efficient if the approximations of the elements of the pairwise comparison matrix made by the ratios of the weights cannot be improved in any position without making it worse in some other position. A pairwise comparison matrix is called double perturbed if it can be made consistent by altering two elements and their reciprocals. The most frequently used weighting method, the eigenvector method is analyzed in the paper, and it is shown that it produces an efficient weight vector for double perturbed pairwise comparison matrices.
\end{abstract}

Keywords: pairwise comparison matrix, efficiency, Pareto optimality, eigenvector

\section{Introduction}

Ranking alternatives, or picking the best alternative is a commonly investigated problem. The case of a single cardinal objective function to be maximized or minimized is long studied by various operations research disciplines. This is however often not feasible. Alternatives can be ranked by assigning a cardinal utility to them, or by setting up ordinal preference relations among them. In the case of a single criterion and a single decision maker, modelling the preferences is often possible through standard methods. If there are multiple, often contradicting criteria, this becomes significantly harder. A dominant alternative, which is the best with respect to all criteria, very rarely exists. Thus, when a decision making method is used to aid the decision of a decision maker, some form of compromise is needed. Modelling the preferences of the decision maker by ranking or weighting the criteria can accomplish such a compromise. It allows the "best" alternative to be chosen (or the possible alternatives to be

\footnotetext{
*E-mail: kristof.abele-nagy@uni-corvinus.hu

${ }^{\dagger}$ E-mail: bozoki.sandor@sztaki.mta.hu

${ }^{\ddagger}$ E-mail: rebakors@gmail.com

${ }^{1}$ Laboratory on Engineering and Management Intelligence, Research Group of Operations Research and Decision Systems, Institute for Computer Science and Control, Hungarian Academy of Sciences; Mail: 1518 Budapest, P.O. Box 63, Hungary.

${ }^{2}$ Department of Operations Research and Actuarial Sciences, Corvinus University of Budapest, Hungary
} 
ranked) with respect to the subjective preferences of the decision maker. Examples of multi-criteria decision problems range from "Which house to buy?" or "What should the company invest in?" to public tenders.

When weighting criteria, giving the weights directly is almost never feasible. Instead, a common method is to apply pairwise comparisons. Answers to the questions "How many times is Criterion A more important than Criterion B?" and so on (which are explicit cardinal ratios) can be arranged in a matrix, called a pairwise comparison matrix (PCM). Formally, a PCM is a square matrix $\mathbf{A}=\left[a_{i j}\right]_{i, j=1, \ldots, n}$ with the properties $a_{i j}>0$ and $a_{i j}=1 / a_{j i}$ (which implies $a_{i i}=1$ ). If the cardinal transitivity property $a_{i k} a_{k j}=a_{i j}$ for all $i, j, k=1, \ldots, n$ also holds for a PCM, it is called consistent, otherwise it is called inconsistent [21]. Let $\mathcal{P C \mathcal { M } _ { n }}$ denote the set of PCMs of size $n \times n$. The next step is to extract the weights of criteria from the PCM. Several methods exist for this task [3, 10, 13, 18]. The eigenvector method (EM) is one of the classical [21, important and most often studied weighting methods related to pairwise comparison matrices, its further analysis is actual and relevant both in decision theory and operations research. We focus on EM in this paper. The eigenvector method gives the weight vector $\mathbf{w}^{E M}=\left(w_{1}, \ldots, w_{n}\right)^{T}$ as the right Perron eigenvector of $\mathbf{A} \in \mathcal{P C} \mathcal{M}_{n}$, thus $\mathbf{A} \mathbf{w}^{E M}=\lambda_{\max } \mathbf{w}^{E M}$ holds, where $\lambda_{\max }$ is the principal eigenvalue of $\mathbf{A} . \lambda_{\max } \geq n$, and $\lambda_{\max }=n$ if and only if $\mathbf{A}$ is consistent [21]. A consistent PCM can be written as

$$
\mathbf{A}=\left(\begin{array}{ccccc}
1 & x_{1} & x_{2} & \ldots & x_{n-1} \\
1 / x_{1} & 1 & x_{2} / x_{1} & \ldots & x_{n-1} / x_{1} \\
1 / x_{2} & x_{1} / x_{2} & 1 & \ldots & x_{n-1} / x_{2} \\
\vdots & \vdots & \vdots & \ddots & \vdots \\
1 / x_{n-1} & x_{1} / x_{n-1} & x_{2} / x_{n-1} & \ldots & 1
\end{array}\right) \in \mathcal{P C} \mathcal{M}_{n}
$$

where $x_{1}, \ldots, x_{n-1}>0$.

The elements of a PCM approximate the ratios of the weights, therefore the ratios of the elements of the weight vector should be as close as possible to the corresponding matrix elements. If a weight vector cannot be trivially improved in this regard (there is no other weight vector which is at least as good approximation, and strictly better in at least one position), it is called Pareto optimal or efficient. It has been proved that the eigenvector method does not always produce an efficient solution [4, Section 3]. However, in some special cases the eigenvector method always gives an efficient weight vector. If the PCM is simple perturbed, i.e., it differs from a consistent PCM in only one element and its reciprocal, the principal right eigenvector is efficient [1]. In the paper this will be extended to double perturbed PCMs, which only differ from a consistent PCM in two elements and their reciprocals.

These special types of PCMs are not just theoretically important, but also occur in real decision problems. Poesz [20] gathered a handful of empirical PCMs that were analyzed in [8]. Although double perturbed PCMs are rare among large PCMs, they appear more frequently among smaller matrices, in other words, when the number of criteria is small. This is especially true if one considers simple perturbed and consistent PCMs as special cases of double perturbed PCMs (see [8, Table 1] - note there is a misprint in the cited Table, the number in the " 3 elements to modify" column in the $4 \times 4$ row should be 6 instead of 0 ). We also conducted an analysis of the prevalence of double perturbed PCMs among the empirical matrices analyzed in [6]. Below is a supplemented version of [8, Table 1] that also includes the results from [6]. The PCMs 


\begin{tabular}{|l|c|c|c|c|c|}
\hline Dimension & $\begin{array}{c}\text { Number of } \\
\text { matrices }\end{array}$ & Consistent & $\begin{array}{c}1 \text { element } \\
\text { to modify }\end{array}$ & $\begin{array}{c}2 \text { elements } \\
\text { to modify }\end{array}$ & $\begin{array}{c}3 \text { elements } \\
\text { to modify }\end{array}$ \\
\hline $3 \times 3$ & 30 & 14 & 16 & - & - \\
\hline $4 \times 4$ & $20+134$ & $1+3$ & $6+10$ & $7+31$ & $6^{*}+90$ \\
\hline $5 \times 5$ & 19 & 1 & 1 & 5 & 1 \\
\hline $6 \times 6$ & $21+152$ & $0+1$ & $1+1$ & $1+1$ & $0+0$ \\
\hline $\begin{array}{l}7 \times 7 \\
\text { and larger }\end{array}$ & $47+159$ & $0+0$ & $1+0$ & $0+0$ & $0+0$ \\
\hline
\end{tabular}

Table 1: The number of element modifications needed to get a consistent PCM.

*There was a misprint in [8, Table 1], this number was 0 . The correct value is 6 .

in [6] had sizes $4 \times 4,6 \times 6$ and $8 \times 8$. The numbers after the + sign are from [6], all others are from [8]. As it can be seen from Table 1, double (and less) perturbed PCMs are rare among large matrices, but they appear among smaller ones.

In Section 2 we will introduce the key definitions and tools used in the paper, together with an example. In Section 3 the main result of the paper is presented: through obtaining explicit formulas for the principal right eigenvector and a series of lemmas, the efficiency of the principal right eigenvector is shown for the case of double perturbed PCMs. The proofs of the lemmas, are given in detail in the Appendix. In Section 4 conclusions follow.

\section{Efficiency and perturbed pairwise comparison ma- trices}

The general form of a multi-objective optimization problem ([14, Chapter 2][23, Chapter 6$]$ ) is

$$
\begin{gathered}
\min \left\{f_{1}(\mathbf{y}), f_{2}(\mathbf{y}), \ldots, f_{m}(\mathbf{y}), \ldots, f_{M}(\mathbf{y})\right\} \\
\text { subject to } \mathbf{y} \in S
\end{gathered}
$$

where $M \geq 2$ denotes the number of objective functions, $f_{m}: \mathbb{R}^{n} \rightarrow \mathbb{R}$ for all $1 \leq m \leq$ $M$. Variables are $\mathbf{y}=\left(y_{1}, y_{2}, \ldots, y_{n}\right)$ and the feasible set is denoted by $S \subseteq \mathbb{R}^{n}$.

Efficiency or Pareto optimality is a basic concept of multi-objective optimization and multi-attribute decision making, too. A vector $\mathbf{y} \in S$ is called efficient, if there does not exist another vector $\mathbf{y}^{\prime} \in S$ such that $f_{m}\left(\mathbf{y}^{\prime}\right) \leq f_{m}(\mathbf{y})$ for all $1 \leq m \leq M$, and $f_{k}\left(\mathbf{y}^{\prime}\right)<f_{k}(\mathbf{y})$ for at least one index $k$.

Let $\mathbf{A}=\left[a_{i j}\right]_{i, j=1, \ldots, n} \in \mathcal{P C} \mathcal{M}_{n}$ and $\mathbf{w}=\left(w_{1}, w_{2}, \ldots, w_{n}\right)^{T}$ be a positive weight vector $\left(S=\mathbb{R}_{++}^{n}\right.$, the positive orthant of the $n$-dimensional Euclidean space), where $n$ is the number of criteria. Let us specify the objective functions by $f_{i j}(\mathbf{w}):=\left|a_{i j}-\frac{w_{i}}{w_{j}}\right|$ for all $i \neq j$. We have $M=n^{2}-n$ objective functions. 
Definition 1. A positive weight vector $\mathbf{w}$ is called efficient if no other positive weight vector $\mathbf{w}^{\prime}=\left(w_{1}^{\prime}, w_{2}^{\prime}, \ldots, w_{n}^{\prime}\right)^{T}$ exists such that

$$
\begin{array}{ll}
\left|a_{i j}-\frac{w_{i}^{\prime}}{w_{j}^{\prime}}\right| \leq\left|a_{i j}-\frac{w_{i}}{w_{j}}\right| & \text { for all } 1 \leq i, j \leq n, \\
\left|a_{k \ell}-\frac{w_{k}^{\prime}}{w_{\ell}^{\prime}}\right|<\left|a_{k \ell}-\frac{w_{k}}{w_{\ell}}\right| & \text { for some } 1 \leq k, \ell \leq n .
\end{array}
$$

A weight vector $\mathbf{w}$ is called inefficient if it is not efficient.

It follows from the definition that an arbitrary renormalization does not influence (in)efficiency.

Remark 1. Weight vector $\mathbf{w}$ is efficient if and only if $c \mathbf{w}$ is efficient for any $c>0$.

For a consistent PCM $a_{i j}=w_{i}^{E M} / w_{j}^{E M}$ for all $i, j=1, \ldots, n$ [21], which implies the following remark:

Remark 2. The principal right eigenvector $\mathbf{w}^{E M}$ is efficient for every consistent PCM.

For inconsistent PCMs however, the principal right eigenvector can be inefficient, found by Blanquero, Carrizosa and Conde [4, Section 3]. This result was also reinforced by Bajwa, Choo and Wedley [3], by Conde and Pérez [11] and by Fedrizzi [17]. Blanquero, Carrizosa and Conde [4] developed LP models to test whether a weight vector is efficient. Bozóki and Fülöp [7] further developed the models and provided algorithms to improve an inefficient weight vector. Anholcer and Fülöp [2] devised a new algorithm to derive an efficient solution from an inconsistent PCM.

Furthermore, Bozóki [5] showed that the principal right eigenvector of a whole class of matrices, namely the parametric PCM

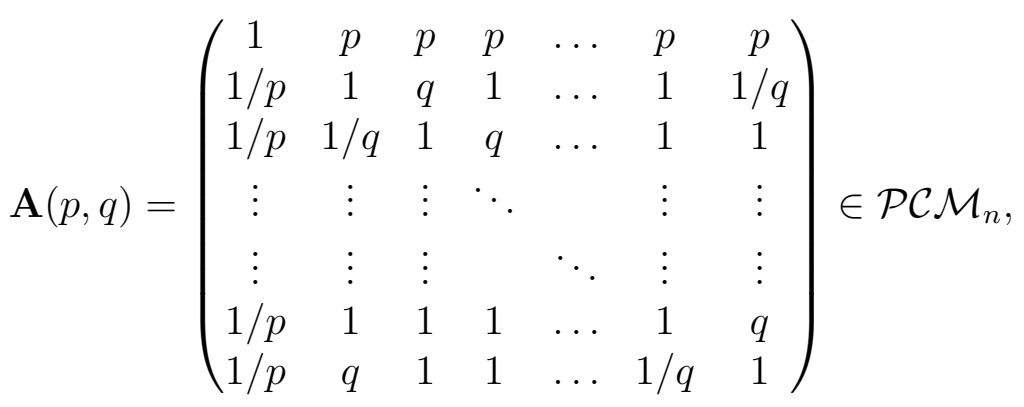

where $n \geq 4, p>0$ and $1 \neq q>0$, is inefficient.

Several necessary and sufficient conditions were examined by Blanquero, Carrizosa and Conde [4, one of which is of crucial importance here. It uses a directed graph representation as follows:

Definition 2. Let $\mathbf{A}=\left[a_{i j}\right]_{i, j=1, \ldots, n} \in \mathcal{P C} \mathcal{M}_{n}$ and $\mathbf{w}=\left(w_{1}, w_{2}, \ldots, w_{n}\right)^{T}$ be a positive weight vector. A directed graph $G=(V, \vec{E})_{\mathbf{A}, \mathbf{w}}$ is defined as follows: $V=\{1,2, \ldots, n\}$ and

$$
\vec{E}=\left\{\operatorname{arc}(i \rightarrow j) \mid \frac{w_{i}}{w_{j}} \geq a_{i j}, i \neq j\right\}
$$

It follows from Definition 2 that if $w_{i} / w_{j}=a_{i j}$, then there is a bidirected arc between nodes $i$ and $j$. The result of Blanquero, Carrizosa and Conde using this representation is as follows: 
Theorem 1 ([4, Corollary 10]). Let $\mathbf{A} \in \mathcal{P C} \mathcal{M}_{n} . A$ weight vector $\mathbf{w}$ is efficient if and only if $G=(V, \vec{E})_{\mathbf{A}, \mathbf{w}}$ is a strongly connected digraph, that is, there exist directed paths from $i$ to $j$ and from $j$ to $i$ for all pairs of nodes $i, j$.

The following numerical example provides an illustration for Theorem 1.

Example 1. Let $\mathbf{A} \in \mathcal{P C} \mathcal{M}_{4}$ be as follows:

$$
\mathbf{A}=\left(\begin{array}{cccc}
1 & 1 & 4 & 7 \\
1 & 1 & 7 & 4 \\
1 / 4 & 1 / 7 & 1 & 3 \\
1 / 7 & 1 / 4 & 1 / 3 & 1
\end{array}\right)
$$

The principal right eigenvector $\mathbf{w}^{E M}$ and the consistent approximation of $\mathbf{A}$ generated by $\mathbf{w}^{E M}$ are as follows:

$$
\mathbf{w}^{E M}=\left(\begin{array}{l}
0.39940672 \\
0.43144159 \\
0.10721105 \\
0.06194064
\end{array}\right), \quad\left[\frac{w_{i}^{E M}}{w_{j}^{E M}}\right]=\left(\begin{array}{cccc}
1 & 0.9257 & 3.7254 & 6.4482 \\
1.0802 & 1 & 4.0242 & 6.9654 \\
0.2684 & 0.2485 & 1 & 1.7309 \\
0.1551 & 0.1436 & 0.5777 & 1
\end{array}\right)
$$

Apply Definition 2, the directed graph $G=(V, \vec{E})_{\mathbf{A}, \mathbf{w}^{E M}}$ corresponding to $\mathbf{A}$ and $\mathbf{w}^{E M}$ is drawn in Figure 1. By Theorem 1, $\mathbf{w}^{E M}$ is not efficient, because the corresponding digraph is not strongly connected: no arc leaves node 1.

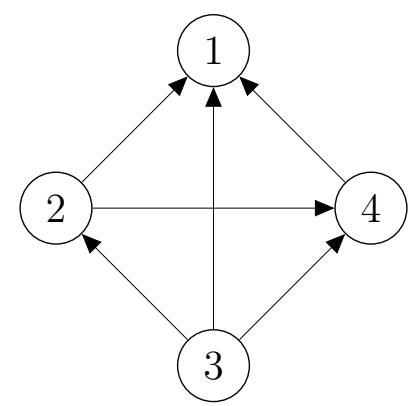

Figure 1: The principal right eigenvector in Example 1 is inefficient, because the corresponding digraph is not strongly connected: no arc leaves node 1

It can be seen in a more constructive way why the principal right eigenvector $\mathbf{w}^{E M}$ is inefficient. Increase the first coordinate until 0.428844188 , and keep the other coordinates unchanged:

$$
\mathbf{w}^{\prime}=\left(\begin{array}{l}
0.428844188 \\
0.431441588 \\
0.107211052 \\
0.061940644
\end{array}\right), \quad\left[\frac{w_{i}^{\prime}}{w_{j}^{\prime}}\right]=\left(\begin{array}{cccc}
1 & \mathbf{0 . 9 9 4 0} & \mathbf{4} & \mathbf{6 . 9 2 3 5} \\
\mathbf{1 . 0 0 6 1} & 1 & 4.0242 & 6.9654 \\
\mathbf{1} / \mathbf{4} & 0.2485 & 1 & 1.7309 \\
\mathbf{0 . 1 4 4 4} & 0.1436 & 0.5777 & 1
\end{array}\right) .
$$

The approximation in the entries marked by bold became strictly better ( $(2)$ holds in Definition 1), while for all other entries the approximation remained the same ((1) 
holds with equality in Definition 1).

As it can be seen from Example 1 above, Theorem 1 is a powerful an applicable characterization of efficiency.

Open problem 1. What is the necessary and sufficient condition of the principal right eigenvector's efficiency?

In the rest of the paper, special types of PCMs are considered.

A simple perturbed PCM differs from a consistent PCM in only one element and its reciprocal, or in other words it can be made consistent by altering only one element (and its reciprocal). Thus, without loss of generality, a simple perturbed PCM can be written as

$$
\mathbf{A}_{\delta}=\left(\begin{array}{ccccc}
1 & \delta x_{1} & x_{2} & \ldots & x_{n-1} \\
1 /\left(\delta x_{1}\right) & 1 & x_{2} / x_{1} & \ldots & x_{n-1} / x_{1} \\
1 / x_{2} & x_{1} / x_{2} & 1 & \ldots & x_{n-1} / x_{2} \\
\vdots & \vdots & \vdots & \ddots & \vdots \\
1 / x_{n-1} & x_{1} / x_{n-1} & x_{2} / x_{n-1} & \ldots & 1
\end{array}\right) \in \mathcal{P C M}_{n}
$$

where $x_{1}, \ldots, x_{n-1}>0$ and $0<\delta \neq 1$.

Theorem 2 ([1, Theorem 3.1]). The principal right eigenvector of a simple perturbed pairwise comparison matrix is efficient.

Similarly, a double perturbed PCM differs from a consistent PCM in two elements and their reciprocals, or in other words it can be made consistent by altering two elements (and their reciprocals). We have to differentiate between three cases of double perturbed PCMs. Without loss of generality, every double perturbed PCM is equivalent to one of them. Also, we can suppose without the loss of generality, that from now on $n \geq 4$, because a PCM with $n=3$ is either simple perturbed or consistent. In Case 1, the perturbed elements are in the same row, and they are multiplied by $0<\delta \neq 1$ and $0<\gamma \neq 1$ respectively. In Case 2, they are in different rows, but this case needs to be further divided into two subcases $(2 \mathrm{~A}$ and $2 \mathrm{~B})$ due to algebraic issues. In Case $2 \mathrm{~A}$ matrix size is $4 \times 4$, while in Case $2 \mathrm{~B}$ matrix size is at least $5 \times 5$. Thus, these matrices take the following form:

Case 1:

$$
\mathbf{P}_{\gamma, \delta}=\left(\begin{array}{cccccc}
1 & \delta x_{1} & \gamma x_{2} & x_{3} & \ldots & x_{n-1} \\
1 /\left(\delta x_{1}\right) & 1 & x_{2} / x_{1} & x_{3} / x_{1} & \ldots & x_{n-1} / x_{1} \\
1 /\left(\gamma x_{2}\right) & x_{1} / x_{2} & 1 & x_{3} / x_{2} & \ldots & x_{n-1} / x_{2} \\
1 / x_{3} & x_{1} / x_{3} & x_{2} / x_{3} & 1 & \ldots & x_{n-1} / x_{3} \\
\vdots & \vdots & \vdots & \vdots & \ddots & \vdots \\
1 / x_{n-1} & x_{1} / x_{n-1} & x_{2} / x_{n-1} & x_{3} / x_{n-1} & \ldots & 1
\end{array}\right)
$$

Case 2A:

$$
\mathbf{Q}_{\gamma, \delta}=\left(\begin{array}{cccc}
1 & \delta x_{1} & x_{2} & x_{3} \\
1 /\left(\delta x_{1}\right) & 1 & x_{2} / x_{1} & x_{3} / x_{1} \\
1 / x_{2} & x_{1} / x_{2} & 1 & \gamma x_{3} / x_{2} \\
1 / x_{3} & x_{1} / x_{3} & x_{2} /\left(\gamma x_{3}\right) & 1
\end{array}\right)
$$


Case 2B:

$$
\mathbf{R}_{\gamma, \delta}=\left(\begin{array}{ccccccc}
1 & \delta x_{1} & x_{2} & x_{3} & x_{4} & \ldots & x_{n-1} \\
1 /\left(\delta x_{1}\right) & 1 & x_{2} / x_{1} & x_{3} / x_{1} & x_{4} / x_{1} & \ldots & x_{n-1} / x_{1} \\
1 / x_{2} & x_{1} / x_{2} & 1 & \gamma x_{3} / x_{2} & x_{4} / x_{2} & \ldots & x_{n-1} / x_{2} \\
1 / x_{3} & x_{1} / x_{3} & x_{2} /\left(\gamma x_{3}\right) & 1 & x_{4} / x_{3} & \ldots & x_{n-1} / x_{3} \\
1 / x_{4} & x_{1} / x_{4} & x_{2} / x_{4} & x_{3} / x_{4} & 1 & \ldots & x_{n-1} / x_{4} \\
\vdots & \vdots & \vdots & \vdots & \vdots & \ddots & \vdots \\
1 / x_{n-1} & x_{1} / x_{n-1} & x_{2} / x_{n-1} & x_{3} / x_{n-1} & x_{4} / x_{n-1} & \ldots & 1
\end{array}\right)
$$

Once again, $x_{1}, \ldots, x_{n-1}>0$ and $0<\delta, \gamma \neq 1$.

Remark 3. If either $\delta=1$ or $\gamma=1$ then the PCM is simple perturbed. If $\delta=\gamma=1$ then the PCM is consistent.

Remark 4. If $n=4$ and $\delta=\gamma$, then the PCM $\mathbf{P}_{\delta, \delta}$ in Case 1 is simple perturbed (multiply the single element $x_{3}$ in position $(1,4)$ by $\delta$ to have a consistent PCM).

Bozóki, Fülöp and Poesz examined PCMs that can be made consistent by modifying at most 3 elements [8]. Each of the three cases above corresponds to a graph: Case 1 corresponds to [8, Fig. 6(b)] while Case 2 corresponds to [8, Fig. 6(a)]. Cook and Kress [12] and Brunelli and Fedrizzi [9] also examined the similar idea of comparing two PCMs that differ in only one element.

\section{Main result: the principal right eigenvector of a double perturbed PCM is efficient}

The main result of the paper is the extension of Theorem 2 for double perturbed PCMs.

Theorem 3. The principal right eigenvector of a double perturbed PCM is efficient.

Proof. For the purpose of easy readability, only an outline of the proof is presented here. The detailed proof can be found in the Appendix.

A method to acquire the explicit form of the principal right eigenvector of a PCM when the perturbed elements are in the same row or column has been developed by Farkas, Rózsa and Stubnya [16]. Farkas [15] writes the explicit formula for the simple perturbed case. Our first goal is to extend the method for the double perturbed case. Similar to [15], the characteristic polynomial is needed first. Proposition 1 covers Case 1 and Proposition 2 covers Cases 2A and 2B.

Using the formulas for the characteristic polynomial, explicit formulas can be derived for the principal right eigenvector. Proposition 3 presents these formulas. For each Case the formulas can be written in several different forms.

Utilizing the different explicit formulas for the principal right eigenvector a series of inequalities can be proved. These inequalities are presented in 28 lemmas (Lemmas 1a $3 \mathrm{~h}$. The different forms of the formula for the principal right eigenvector make it possible to use the form most suited to each proof. Obtaining these inequalities makes it possible to prove the efficiency of the principal right eigenvector of a double perturbed PCM. 
As per Theorem 1, the strong connectedness of the digraph in Definition 2 needs to be shown. All possible digraphs are shown in Figures 24. The direction of each arc (where applicable) is determined by the corresponding Lemma using Definition 2 , which is labeled on the arc itself. In the cases where there is a node named $i$, this represents the complete subgraph of the rest of the nodes (consisting of $n-3$ in Case 1 and $n-4$ nodes in Case 2B). In these subgraphs there are bidirected arcs between any two nodes, due to Lemmas $1 \mathrm{j}]$ and $3 \mathrm{~h}$. This is a strongly connected subgraph, and for any fixed $j \leq 3$ the direction of the arc between nodes $i$ and $j$ is the same for every $i \geq 4$ in Case 1 (see Lemmas 1e, 1f, 1h, 1i). Similarly, for any fixed $j \leq 4$ the direction of the arc between nodes $i$ and $j$ is the same for every $i \geq 5$ in Case 2B (see Lemmas $3 \mathrm{c}, 3 \mathrm{~d}, 3 \mathrm{e}$. Hence, it can be contracted into a single node when analyzing strong connectedness. Figures 2, 3, 4 correspond to Cases 1, 2A, 2B respectively. For the strong connectedness of each digraph, it is sufficient to find a directed cycle. Unchecked arcs are denoted by dashed lines in Figures 2 4. The directed cycles are presented in Corollary 1, 2 and 3 for Cases 1,2A and 2B respectively.

The presence of a directed cycle implies strong connectedness for all of the digraphs, which implies efficiency in all cases by Theorem 1 .

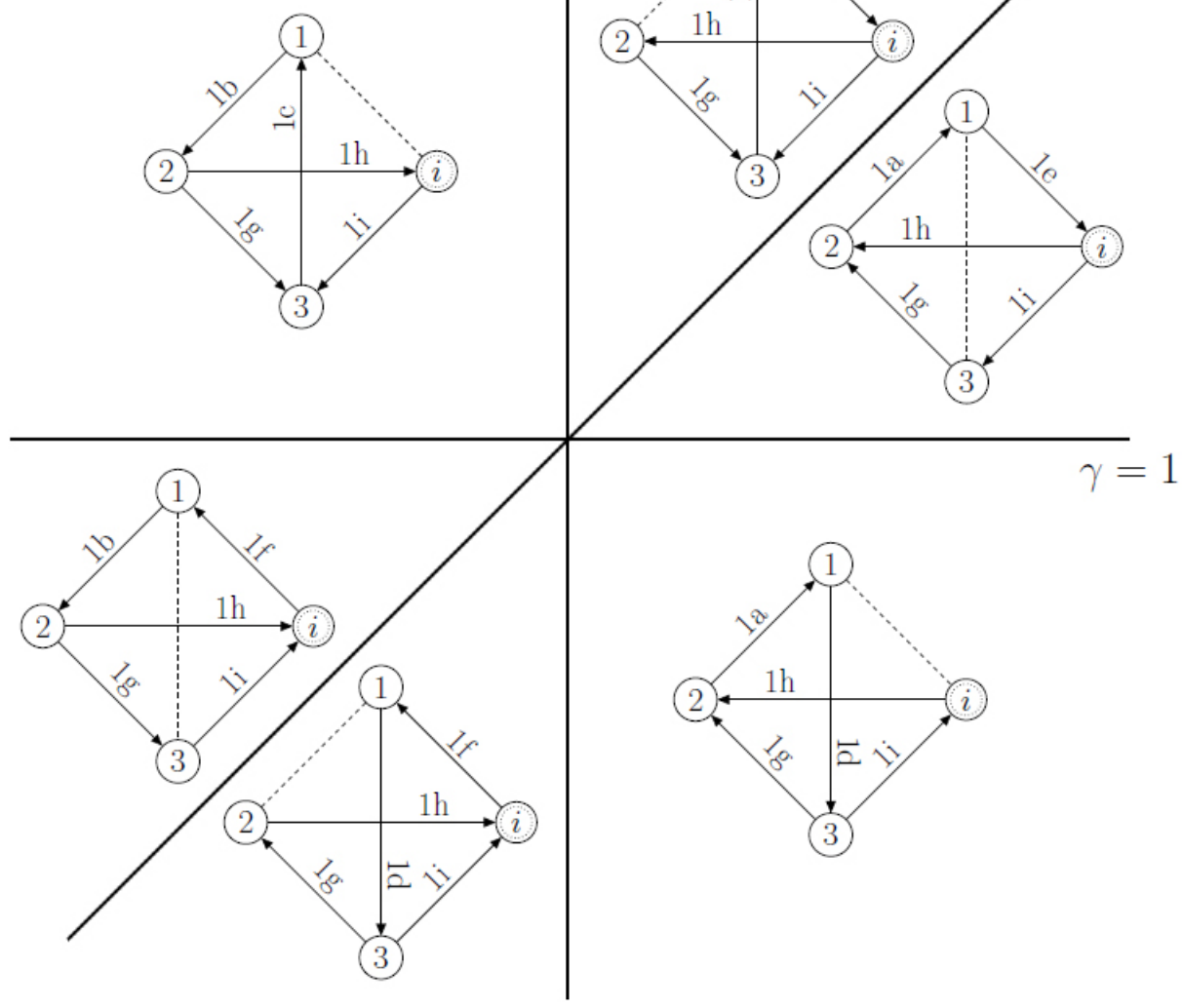

Figure 2: The digraph of the principal right eigenvector in Case 1 is strongly connected, independently of the orientation of dashed arcs that have not been analyzed 


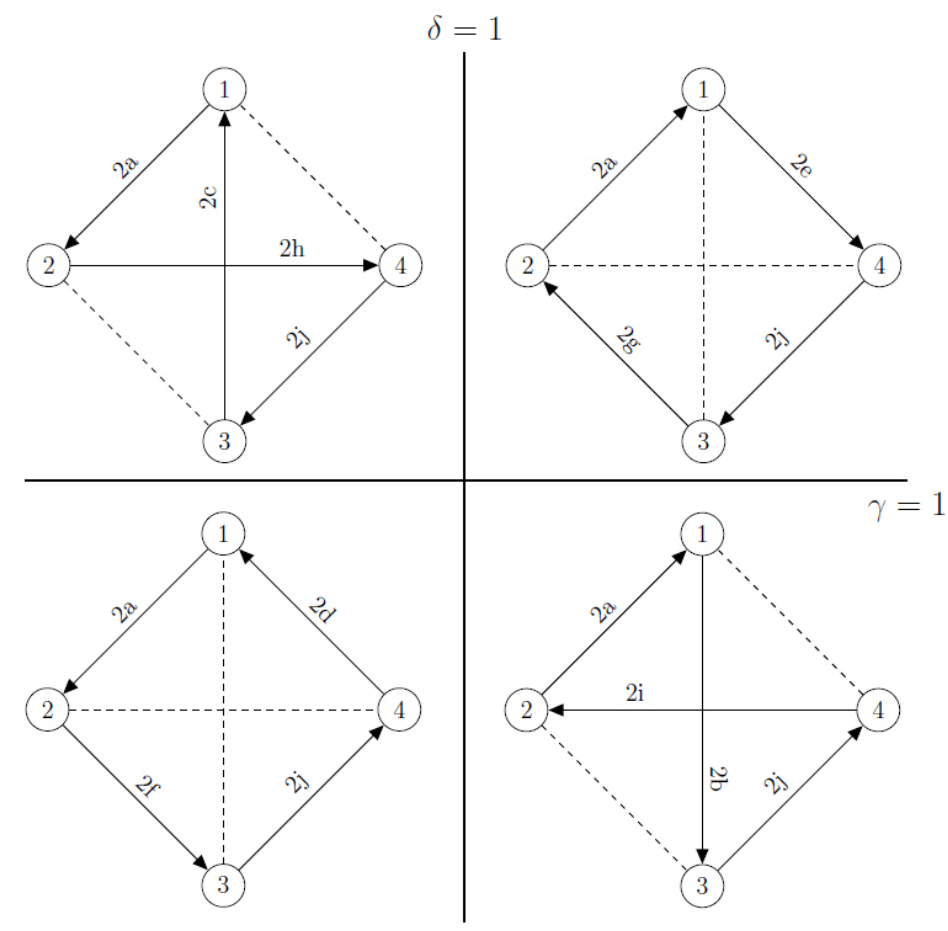

Figure 3: The digraph of the principal right eigenvector in Case 2A is strongly connected, independently of the orientation of dashed arcs that have not been analyzed

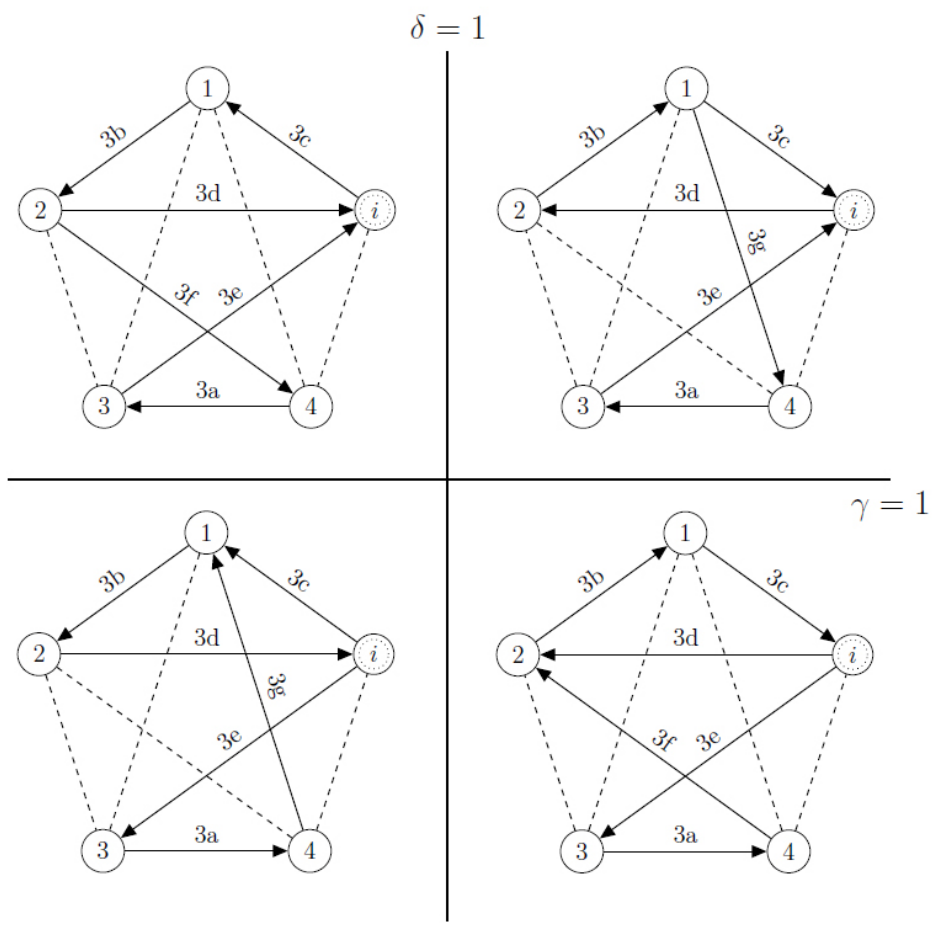

Figure 4: The digraph of the principal right eigenvector in Case 2B is strongly connected, independently of the orientation of dashed arcs that have not been analyzed 


\section{Conclusions}

In the paper we used linear algebraic methods to derive explicit formulas for the principal eigenvector of double perturbed PCMs. We also used a necessary and sufficient condition for efficiency which uses a directed graph representation (the weight vector is efficient if and only if this graph is strongly connected) developed by Blanquero, Carrizosa and Conde [4. Double perturbed PCMs had to be divided into three cases in order to get explicit formulas for every case. In all three cases the digraph has been studied arc by arc, however not all arcs had to be studied in order to determine strong connectedness. Utilizing all these tools, we have shown in the paper, that the often used eigenvector method produces an efficient weight vector in the case of double perturbed PCMs. This is an extension of our earlier result for simple perturbed PCMs [1].

A direct extension to the triple (or more) perturbed case is not possible, since all PCMs of at least $4 \times 4$ size which are not (at most) double perturbed are triple perturbed, and there are examples, e.g. Example 1, of inefficiency of size $4 \times 4$. Thus, while in some cases (e.g. when all perturbed elements are in different rows/columns) it may be possible to show efficiency, for all triple perturbed PCMs this is impossible. Furthermore, a triple perturbed PCM can be equivalent to five separate basic cases (see [8, Fig. 7]), which may need to be further divided into more subcases, making the efficiency analysis of triple perturbed PCMs difficult. A full characterization of the efficiency of the principal right eigenvector is still an open question, and a possible subject of future research.

\section{Acknowledgements}

The authors are grateful to the anonymous reviewers for their constructive remarks. András Farkas (Óbuda University, Budapest) and János Fülöp (Institute for Computer Science and Control, Hungarian Academy of Sciences (MTA SZTAKI) and Óbuda University, Budapest) are greatly acknowledged for their valuable comments. Research was supported in part by the Hungarian Scientific Research Fund (OTKA) grant K 111797. S. Bozóki acknowledges the support of the János Bolyai Research Fellowship no. $\mathrm{BO} / 00154 / 16 / 3$.

\section{References}

[1] K. Ábele-Nagy and S. Bozóki. Efficiency analysis of simple perturbed pairwise comparison matrices. Fundamenta Informaticae, 144(3-4):279-289, 2016.

[2] M. Anholcer and J. Fülöp. Deriving priorities from inconsistent PCM using the network algorithms. Under review, http://arxiv.org/pdf/1510.04315, 2017.

[3] G. Bajwa, E. U. Choo, and W. C. Wedley. Effectiveness analysis of deriving priority vectors from reciprocal pairwise comparison matrices. Asia-Pacific Journal of Operational Research, 25(3):279-299, 2008. 
[4] R. Blanquero, E. Carrizosa, and E. Conde. Inferring efficient weights from pairwise comparison matrices. Mathematical Methods of Operations Research, 64(2):271-284, 2006.

[5] S. Bozóki. Inefficient weights from pairwise comparison matrices with arbitrarily small inconsistency. Optimization, 63(12):1893-1901, 2014.

[6] S. Bozóki, L. Dezsô, A. Poesz, and J. Temesi. Analysis of pairwise comparison matrices: an empirical research. Annals of Operations Research, 211(1):511-528, feb 2013.

[7] S. Bozóki and J. Fülöp. Efficient weight vectors from pairwise comparison matrices. European Journal of Operational Research, 264(2):419-427, 2018.

[8] S. Bozóki, J. Fülöp, and A. Poesz. On pairwise comparison matrices that can be made consistent by the modification of a few elements. Central European Journal of Operations Research, 19(2):157-175, 2011.

[9] M. Brunelli and M. Fedrizzi. Axiomatic properties of inconsistency indices for pairwise comparisons. Journal of the Operational Research Society, 66(1):1-15, 2014.

[10] E. Choo and W. Wedley. A common framework for deriving preference values from pairwise comparison matrices. Computers \& Operations Research, 31(6):893-908, 2004.

[11] E. Conde and M. d. 1. P. R. Pérez. A linear optimization problem to derive relative weights using an interval judgement matrix. European Journal of Operational Research, 201(2):537-544, 2010.

[12] W. Cook and M. Kress. Deriving weights from pairwise comparison ratio matrices: An axiomatic approach. European Journal of Operational Research, 37(3):355362, 1988.

[13] T. K. Dijkstra. On the extraction of weights from pairwise comparison matrices. Central European Journal of Operations Research, 21(1):103-123, 2013.

[14] M. Ehrgott. Multicriteria Optimization. Volume 491 of Lecture Notes in Economics and Mathematical Systems. Springer Verlag, Berlin, 2000.

[15] A. Farkas. The analysis of the principal eigenvector of pairwise comparison matrices. Acta Polytechnica Hungarica, 4(2):99-115, 2007.

[16] A. Farkas, P. Rózsa, and E. Stubnya. Transitive matrices and their applications. Linear Algebra and its Applications, 302-303:423-433, 1999.

[17] M. Fedrizzi. Obtaining non-dominated weights from preference relations through norm-induced distances. XXXVII Meeting of the Italian Association for Mathematics Applied to Economic and Social Sciences (AMASES), September 5-7, 2013, Stresa, Italy, 2013. 
[18] B. Golany and M. Kress. A multicriteria evaluation of methods for obtaining weights from ratio-scale matrices. European Journal of Operational Research, 69(2):210-220, 1993.

[19] D. A. Harville. Matrix Algebra From a Statistician's Perspective. Springer, 2008.

[20] A. Poesz. Empirical pairwise comparison matrices (EPCM) - an on-line collection from real decisions, version EPCM-October-2009. http://www.sztaki.hu/\%7ebozoki/epcm, 2009.

[21] T. L. Saaty. A scaling method for priorities in hierarchical structures. Journal of Mathematical Psychology, 15(3):234-281, 1977.

[22] J. Sherman and W. J. Morrison. Adjustment of an inverse matrix corresponding to a change in one element of a given matrix. Annals of Mathematical Statistics, 21(1):124-127, 1950.

[23] R. E. Steuer. Multiple Criteria Optimization: Theory, Computation, and Application. Wiley Series in Probability and Mathematical Statistics. Wiley, 1986. 


\section{Appendix}

Let $\mathbf{D}=\operatorname{diag}\left(1,1 / x_{1}, \ldots, 1 / x_{n-1}\right)$, and let $\mathbf{e}=(1, \ldots, 1)^{T}$. For $\mathbf{A} \in \mathcal{P C} \mathcal{M}_{n}$,

$$
\mathbf{e e}^{T}-\mathbf{U}_{i} \mathbf{V}_{i}^{T}=\mathbf{D}^{-1} \mathbf{A D}
$$

holds for $i=1,2$. For $i=1$, A has form (3) (Case 1) and

$$
\mathbf{U}_{1}=\left(\begin{array}{cc}
0 & 1 \\
1-1 / \delta & 0 \\
1-1 / \gamma & 0 \\
0 & 0 \\
\vdots & \vdots \\
0 & 0
\end{array}\right) \in \mathbb{R}^{n \times 2}, \quad \mathbf{V}_{1}=\left(\begin{array}{cc}
1 & 0 \\
0 & 1-\delta \\
0 & 1-\gamma \\
0 & 0 \\
\vdots & \vdots \\
0 & 0
\end{array}\right) \in \mathbb{R}^{n \times 2}
$$

For $i=2$, A has form (4) or (5) (Case 2) and

$$
\mathbf{U}_{2}=\left(\begin{array}{cccc}
0 & 1 & 0 & 0 \\
1-1 / \delta & 0 & 0 & 0 \\
0 & 0 & 0 & 1 \\
0 & 0 & 1-1 / \gamma & 0 \\
0 & 0 & 0 & 0 \\
\vdots & \vdots & \vdots & \vdots \\
0 & 0 & 0 & 0
\end{array}\right) \in \mathbb{R}^{n \times 4}, \mathbf{V}_{2}=\left(\begin{array}{cccc}
1 & 0 & 0 & 0 \\
0 & 1-\delta & 0 & 0 \\
0 & 0 & 1 & 0 \\
0 & 0 & 0 & 1-\gamma \\
0 & 0 & 0 & 0 \\
\vdots & \vdots & \vdots & \vdots \\
0 & 0 & 0 & 0
\end{array}\right) \in \mathbb{R}^{n \times 4}
$$

Lemma 1 (Matrix determinant lemma, [19]). If $\mathbf{A} \in \mathbb{R}^{n \times n}$ is invertible, and $\mathbf{U}, \mathbf{V} \in$ $\mathbb{R}^{n \times m}$, then

$$
\operatorname{det}\left(\mathbf{A}+\mathbf{U V}^{T}\right)=\operatorname{det}\left(\mathbf{I}_{m}+\mathbf{V}^{T} \mathbf{A}^{-1} \mathbf{U}\right) \operatorname{det}(\mathbf{A}),
$$

where $\mathbf{I}_{m}$ denotes the identity matrix of size $m \times m$.

Lemma 2 (Sherman-Morrison formula, [22]). Let $\mathbf{A} \in \mathbb{R}^{n \times n}, \mathbf{u}, \mathbf{v} \in \mathbb{R}^{n}$. If $\mathbf{A}$ is invertible and $1+\mathbf{v}^{T} \mathbf{A}^{-1} \mathbf{u} \neq 0$, then $\left(\mathbf{A}+\mathbf{u v}^{T}\right)^{-1}$ exists, and

$$
\left(\mathbf{A}+\mathbf{u} \mathbf{v}^{T}\right)^{-1}=\mathbf{A}^{-1}-\frac{1}{1+\mathbf{v}^{T} \mathbf{A}^{-1} \mathbf{u}} \mathbf{A}^{-1} \mathbf{u v}^{T} \mathbf{A}^{-1} .
$$

Let $\mathbf{A} \in \mathcal{P C} \mathcal{M}_{n}$ be a double perturbed PCM and $\mathbf{U}_{i}, \mathbf{V}_{i}$ be as in (6). Let the matrix $\mathbf{K}_{\mathbf{A}}(\lambda) \in \mathbb{R}^{n \times n}$ be defined as follows:

$$
\mathbf{K}_{\mathbf{A}}(\lambda)=\lambda \mathbf{I}+\mathbf{U}_{i} \mathbf{V}_{i}^{T}-\mathbf{e e}^{T}=\lambda \mathbf{I}-\mathbf{D}^{-1} \mathbf{A D},
$$

where $\mathbf{I}$ denotes $\mathbf{I}_{n}, \mathbf{e}=(1, \ldots, 1)^{T} \in \mathbb{R}^{n}, i=1$ in Case $1, i=2$ in Case 2, and the second equation follows from (6).

Lemma 3. The characteristic polynomial of the double perturbed $P C M \mathbf{A} \in \mathcal{P C} \mathcal{M}_{n}$ is

$$
p_{\mathbf{A}}(\lambda)=(-1)^{n} \operatorname{det}\left(\mathbf{K}_{\mathbf{A}}(\lambda)\right)
$$


Proof. As before, $i=1$ in Case 1 and $i=2$ in Case 2.

$$
\begin{aligned}
p_{\mathbf{A}}(\lambda) & =\operatorname{det}(\mathbf{A}-\lambda \mathbf{I}) \\
& =(-1)^{n} \operatorname{det}(\lambda \mathbf{I}-\mathbf{A}) \\
& =(-1)^{n} \operatorname{det}\left(\lambda \mathbf{I}+\mathbf{D}\left(\mathbf{U}_{i} \mathbf{V}_{i}^{T}-\mathbf{e e}^{T}\right) \mathbf{D}^{-1}\right) \\
& =(-1)^{n} \operatorname{det}\left(\mathbf{D}\left(\lambda \mathbf{I}+\mathbf{U}_{i} \mathbf{V}_{i}^{T}-\mathbf{e e}^{T}\right) \mathbf{D}^{-1}\right) \\
& =(-1)^{n} \operatorname{det}(\mathbf{D}) \operatorname{det}\left(\lambda \mathbf{I}+\mathbf{U}_{i} \mathbf{V}_{i}^{T}-\mathbf{e e}^{T}\right) \operatorname{det}\left(\mathbf{D}^{-1}\right) \\
& =(-1)^{n} \operatorname{det}\left(\mathbf{K}_{\mathbf{A}}(\lambda)\right) .
\end{aligned}
$$

Lemma 4. $\operatorname{det}\left(\lambda \mathbf{I}-\mathbf{e e}^{T}\right)=\lambda^{n}-n \lambda^{n-1}$.

Proof. If $\lambda=0$, then both sides of the equation are 0 . If $\lambda \neq 0$, apply Lemma 1 with $m=1, \mathbf{A}=\lambda \mathbf{I}, \mathbf{U}=-\mathbf{e}, \mathbf{V}=\mathbf{e}:$

$$
\operatorname{det}\left(\lambda \mathbf{I}-\mathbf{e e}^{T}\right)=\left(1-\mathbf{e}^{T}(\lambda \mathbf{I})^{-1} \mathbf{e}\right) \operatorname{det}(\lambda \mathbf{I})=\lambda^{n}-n \lambda^{n-1} .
$$

Lemma 5. If $\lambda \neq 0$ and $\lambda \neq n$, then $\left(\lambda \mathbf{I}-\mathbf{e e}^{T}\right)^{-1}$ exists, and

$$
\left(\lambda \mathbf{I}-\mathbf{e e}^{T}\right)^{-1}=\frac{1}{\lambda(\lambda-n)} \mathbf{e e}^{T}+\frac{1}{\lambda} \mathbf{I} .
$$

Proof. Apply the Sherman-Morrison formula (Lemma 2) with $\mathbf{A}=\lambda \mathbf{I}, \mathbf{u}=-\mathbf{e}$, $\mathbf{v}=\mathbf{e}$.

Lemma 6. Let $\mathbf{U}, \mathbf{V} \in \mathbb{R}^{n \times m}$ be arbitrary matrices. If $\lambda \neq 0$ and $\lambda \neq n$, then

$$
\operatorname{det}\left(\lambda \mathbf{I}_{n}+\mathbf{U} \mathbf{V}^{T}-\mathbf{e e}^{T}\right)=\left(\lambda^{n}-n \lambda^{n-1}\right) \operatorname{det}\left(\mathbf{I}_{m}+\frac{1}{\lambda(\lambda-n)} \mathbf{V}^{T} \mathbf{e e}^{T} \mathbf{U}+\frac{1}{\lambda} \mathbf{V}^{T} \mathbf{U}\right) .
$$

Proof. Apply Lemma 1 with $\mathbf{A}=\lambda \mathbf{I}_{n}-\mathbf{e e}^{T}$. According to Lemma 5, A is invertible. Utilizing Lemmas 1, 4 and 5 the following equations hold:

$$
\begin{aligned}
& \operatorname{det}\left(\left(\lambda \mathbf{I}_{n}-\mathbf{e e}^{T}\right)+\mathbf{U V}^{T}\right) \\
& =\operatorname{det}\left(\mathbf{I}_{m}+\mathbf{V}^{T}\left(\lambda \mathbf{I}_{n}-\mathbf{e} \mathbf{e}^{T}\right)^{-1} \mathbf{U}\right) \operatorname{det}\left(\lambda \mathbf{I}_{n}-\mathbf{e e}^{T}\right) \\
& =\operatorname{det}\left(\mathbf{I}_{m}+\frac{1}{\lambda(\lambda-n)} \mathbf{V}^{T} \mathbf{e} \mathbf{e}^{T} \mathbf{U}+\frac{1}{\lambda} \mathbf{V}^{T} \mathbf{U}\right)\left(\lambda^{n}-n \lambda^{n-1}\right) .
\end{aligned}
$$
form.

We can write the characteristic polynomial of double perturbed PCMs in explicit

Proposition 1. Let $n \geq 4$. The characteristic polynomial of a double perturbed PCM in form (3) (Case 1) is

$$
p_{\mathbf{P}}(\lambda)=(-1)^{n} \lambda^{n-3}\left(\lambda^{3}-n \lambda^{2}-\left(\frac{\gamma}{\delta}+\frac{\delta}{\gamma}\right)-(n-3)\left(\gamma+\delta+\frac{1}{\gamma}+\frac{1}{\delta}\right)+4 n-10\right) .
$$


Proof. Lemma 3 implies that

$$
p_{\mathbf{P}}(\lambda)=(-1)^{n} \operatorname{det}\left(\mathbf{K}_{\mathbf{P}}(\lambda)\right)=(-1)^{n} \operatorname{det}\left(\lambda \mathbf{I}+\mathbf{U}_{1} \mathbf{V}_{1}^{T}-\mathbf{e e}^{T}\right) \text {, }
$$

where $\mathbf{U}_{1}$ and $\mathbf{V}_{1}$ are defined by (7). Suppose that $\lambda \neq n$ and $\lambda \neq 0$. According to Lemma 6

$$
\begin{aligned}
p_{\mathbf{P}}(\lambda) & =(-1)^{n}\left(\lambda^{n}-n \lambda^{n-1}\right) \operatorname{det}\left(\mathbf{I}_{2}+\frac{1}{\lambda(\lambda-n)} \mathbf{V}_{1}^{T} \mathbf{e e}^{T} \mathbf{U}_{1}+\frac{1}{\lambda} \mathbf{V}_{1}^{T} \mathbf{U}_{1}\right) \\
& =(-1)^{n}\left(\lambda^{n}-n \lambda^{n-1}\right) \operatorname{det}(\mathbf{S}) \\
& =(-1)^{n} \lambda^{n-3}\left(\lambda^{3}-n \lambda^{2}-\left(\frac{\gamma}{\delta}+\frac{\delta}{\gamma}\right)-(n-3)\left(\gamma+\delta+\frac{1}{\gamma}+\frac{1}{\delta}\right)+4 n-10\right),
\end{aligned}
$$

where

$$
\mathbf{S}=\left(\begin{array}{cc}
1+\frac{2-1 / \delta-1 / \gamma}{\lambda(\lambda-n)} & \frac{1}{\lambda(\lambda-n)}+\frac{1}{\lambda} \\
\frac{(2-\delta-\gamma)(1-1 / \delta)+(2-\delta-\gamma)(1-1 / \gamma)}{\lambda(\lambda-n)}+\frac{(1-\delta)(1-1 / \delta)}{\lambda}+\frac{(1-\gamma)(1-1 / \gamma)}{\lambda} & 1+\frac{2-\delta-\gamma}{\lambda(\lambda-n)}
\end{array}\right) .
$$

A polynomial of degree $n$ is uniquely determined by $n+1$ points, and we have calculated $p_{\mathbf{P}}(\lambda)$ in all but two points, which completes the proof.

Proposition 2. Let $n \geq 4$. The characteristic polynomial of a double perturbed PCM in form (5) (Case 2B) is

$$
p_{\mathbf{R}}(\lambda)=(-1)^{n} \lambda^{n-5}\left(\lambda^{5}-n \lambda^{4}-(n-2)\left(\gamma+\delta+\frac{1}{\gamma}+\frac{1}{\delta}-4\right) \lambda^{2}-c \lambda-(n-4) c\right)
$$

where

$$
c=\frac{(\gamma-1)^{2}(\delta-1)^{2}}{\gamma \delta} .
$$

Furthermore, the characteristic polynomial of a double perturbed PCM in form (4) (Case 2A), $p_{\mathbf{Q}}(\lambda)$ is a special case of $p_{\mathbf{R}}(\lambda)$ with $n=4$. Namely,

$$
p_{\mathbf{Q}}(\lambda)=\lambda^{4}-4 \lambda^{3}-2\left(\gamma+\delta+\frac{1}{\gamma}+\frac{1}{\delta}-4\right) \lambda-\frac{(\gamma-1)^{2}(\delta-1)^{2}}{\gamma \delta} .
$$

Proof. Lemma 3 implies that

$$
p_{\mathbf{R}}(\lambda)=(-1)^{n} \operatorname{det}\left(\mathbf{K}_{\mathbf{R}}(\lambda)\right)=(-1)^{n} \operatorname{det}\left(\lambda \mathbf{I}+\mathbf{U}_{2} \mathbf{V}_{2}^{T}-\mathbf{e e}^{T}\right),
$$

where $\mathbf{U}_{2}$ and $\mathbf{V}_{2}$ are defined by (8). Suppose that $\lambda \neq n$ and $\lambda \neq 0$. According to Lemma 6

$$
\begin{aligned}
p_{\mathbf{R}}(\lambda) & =(-1)^{n}\left(\lambda^{n}-n \lambda^{n-1}\right) \operatorname{det}\left(\mathbf{I}_{4}+\frac{1}{\lambda(\lambda-n)} \mathbf{V}_{2}^{T} \mathbf{e e}^{T} \mathbf{U}_{2}+\frac{1}{\lambda} \mathbf{V}_{2}^{T} \mathbf{U}_{2}\right) \\
& =(-1)^{n}\left(\lambda^{n}-n \lambda^{n-1}\right) \operatorname{det}(\mathbf{T}) \\
& =(-1)^{n} \lambda^{n-5}\left(\lambda^{5}-n \lambda^{4}-(n-2)\left(\gamma+\delta+\frac{1}{\gamma}+\frac{1}{\delta}-4\right) \lambda^{2}-c \lambda-(n-4) c\right),
\end{aligned}
$$


where

$$
\mathbf{T}=\left(\begin{array}{cccc}
1+\frac{1-1 / \delta}{\lambda(\lambda-n)} & \frac{1}{\lambda(\lambda-n)}+\frac{1}{\lambda} & \frac{1-1 / \gamma}{\lambda(\lambda-n)} & \frac{1}{\lambda(\lambda-n)} \\
\frac{(1-\delta)(1-1 / \delta)}{\lambda(\lambda-n)}+\frac{(1-\delta)(1-1 / \delta)}{\lambda} & 1+\frac{1-\delta}{\lambda(\lambda-n)} & \frac{(1-\delta)(1-1 / \gamma)}{\lambda(\lambda-n)} & \frac{1-\delta}{\lambda(\lambda-n)} \\
\frac{1-1 / \delta}{\lambda(\lambda-n)} & \frac{1}{\lambda(\lambda-n)} & 1+\frac{1-1 / \gamma}{\lambda(\lambda-n)} & \frac{1}{\lambda(\lambda-n)}+\frac{1}{\lambda} \\
\frac{(1-\gamma)(1-1 / \delta)}{\lambda(\lambda-n)} & \frac{1-\gamma}{\lambda(\lambda-n)} & \frac{(1-\gamma)(1-1 / \gamma)}{\lambda(\lambda-n)}+\frac{(1-\gamma)(1-1 / \gamma)}{\lambda} & 1+\frac{1-\gamma}{\lambda(\lambda-n)}
\end{array}\right)
$$

and

$$
c=\frac{(\gamma-1)^{2}(\delta-1)^{2}}{\gamma \delta}
$$

Again, a polynomial of degree $n$ is uniquely determined by $n+1$ points, and we have calculated $p_{\mathbf{R}}(\lambda)$ in all but two points, which completes the proof. The case $n=4$ is analogous, and

$$
p_{\mathbf{Q}}(\lambda)=\lambda^{4}-4 \lambda^{3}-2\left(\gamma+\delta+\frac{1}{\gamma}+\frac{1}{\delta}-4\right) \lambda-\frac{(\gamma-1)^{2}(\delta-1)^{2}}{\gamma \delta}
$$

is resulted in.

Proposition 3. The principle right eigenvector of a double perturbed PCM can be written in explicit ways.

In Case 1 ( $\gamma$ and $\delta$ are in the same row), the formulas for the principal right eigenvector are the following:

$$
\mathbf{w}^{E M}=\left(\begin{array}{c}
\delta \gamma \lambda(\lambda-n+1) \\
\mathbf{w}^{E M}=\left(\begin{array}{c}
\frac{1}{x_{1}}[\gamma \lambda-(n-2) \gamma+\delta+(n-3) \delta \gamma] \\
\frac{1}{x_{2}}[\delta \lambda-(n-2) \delta+\gamma+(n-3) \delta \gamma] \\
\frac{1}{x_{3}}[\gamma+\delta+\delta \gamma \lambda-2 \delta \gamma] \\
\vdots \\
\frac{1}{x_{i-1}}[\gamma+\delta+\delta \gamma \lambda-2 \delta \gamma] \\
\vdots \\
\frac{1}{x_{n-1}}[\gamma+\delta+\delta \gamma \lambda-2 \delta \gamma] \\
x_{1} \gamma \lambda[\delta \lambda-(n-2) \delta+\gamma+n-3] \\
\gamma \lambda^{3}-(n-1) \gamma \lambda^{2}-(n-3)\left(\gamma^{2}-2 \gamma+1\right) \\
\frac{x_{1}}{x_{2}}\left[\gamma \lambda^{2}-\gamma \lambda+\delta \lambda+(n-3)(\delta \gamma-\delta-\gamma+1)\right] \\
\frac{x_{1}}{x_{3}}\left[\gamma \lambda^{2}-\gamma \lambda-\gamma+\delta+\delta \gamma \lambda-\delta \gamma+\gamma^{2}\right] \\
\vdots \\
\frac{x_{1}}{x_{i-1}}\left[\gamma \lambda^{2}-\gamma \lambda-\gamma+\delta+\delta \gamma \lambda-\delta \gamma+\gamma^{2}\right] \\
\vdots \\
\frac{x_{1}}{x_{n-1}}\left[\gamma \lambda^{2}-\gamma \lambda-\gamma+\delta+\delta \gamma \lambda-\delta \gamma+\gamma^{2}\right]
\end{array}\right),
\end{array}\right.
$$




$$
\mathbf{w}^{E M}=\left(\begin{array}{c}
x_{2} \delta \lambda[\delta+\gamma \lambda-(n-2) \gamma+n-3] \\
\frac{x_{2}}{x_{1}}\left[\delta \lambda^{2}-\delta \lambda+\gamma \lambda+(n-3)(\delta \gamma-\delta-\gamma+1)\right] \\
\delta \lambda^{3}-(n-1) \delta \lambda^{2}-(n-3)\left(\delta^{2}-2 \delta+1\right) \\
\frac{x_{2}}{x_{3}}\left[\delta \lambda^{2}-\delta \lambda+\gamma-\delta+\delta^{2}+\delta \gamma \lambda-\delta \gamma\right] \\
\vdots \\
\frac{x_{2}}{x_{i-1}}\left[\delta \lambda^{2}-\delta \lambda+\gamma-\delta+\delta^{2}+\delta \gamma \lambda-\delta \gamma\right] \\
\vdots \\
\frac{x_{2}}{x_{n-1}}\left[\delta \lambda^{2}-\delta \lambda+\gamma-\delta+\delta^{2}+\delta \gamma \lambda-\delta \gamma\right] \\
x_{3} \delta \gamma \lambda(\delta+\gamma+\lambda-2) \\
\mathbf{w}^{E M}=\left(\begin{array}{c}
x_{3}\left[\delta \gamma \lambda^{2}-\delta \gamma \lambda+\gamma^{2}+\gamma \lambda-\gamma-\delta \gamma+\delta\right] \\
\frac{x_{3}}{x_{2}}\left[\delta \gamma \lambda^{2}-\delta \gamma \lambda-\delta \gamma+\gamma+\delta^{2}+\delta \lambda-\delta\right] \\
\delta \gamma \lambda^{2}-4 \delta \gamma+\gamma+\delta+\delta^{2} \gamma+\gamma^{2} \delta \\
\frac{x_{3}}{x_{4}}\left[\delta \gamma \lambda^{2}-4 \delta \gamma+\gamma+\delta+\delta^{2} \gamma+\gamma^{2} \delta\right] \\
\vdots \\
\frac{x_{3}}{x_{n-1}}\left[\delta \gamma \lambda^{2}-4 \delta \gamma+\gamma+\delta+\delta^{2} \gamma+\gamma^{2} \delta\right]
\end{array}\right)
\end{array}\right.
$$

Formulas (9)-(12) give the same principal right eigenvector, up to a scalar multiplier.

In Case $2 A(\gamma$ and $\delta$ are in different rows, and matrix size is $4 \times 4)$ the formulas take the following form:

$$
\begin{aligned}
& \mathbf{w}^{E M}=\left(\begin{array}{c}
\delta\left(\lambda^{3} \gamma-3 \lambda^{2} \gamma-1+2 \gamma-\gamma^{2}\right) \\
\frac{1}{x_{1}}\left[\lambda^{2} \gamma-2 \lambda \gamma+\delta+2 \lambda \delta \gamma-2 \delta \gamma+\delta \gamma^{2}\right] \\
\frac{1}{x_{2}} \gamma\left[\gamma+\lambda-1+\delta \lambda^{2}-2 \lambda \delta+\delta+\lambda \delta \gamma-\delta \gamma\right] \\
\frac{1}{x_{3}}\left[1+\lambda \gamma-\gamma+\lambda \delta-\delta+\delta \gamma \lambda^{2}-2 \lambda \delta \gamma+\delta \gamma\right]
\end{array}\right), \\
& \mathbf{w}^{E M}=\left(\begin{array}{c}
x_{1}\left[\delta \gamma \lambda^{2}-2 \lambda \delta \gamma+1+2 \lambda \gamma-2 \gamma+\gamma^{2}\right] \\
\lambda^{3} \gamma-3 \lambda^{2} \gamma-1+2 \gamma-\gamma^{2} \\
\frac{x_{1}}{x_{2}} \gamma\left[\lambda \gamma+\lambda^{2}-2 \lambda-\gamma+1+\lambda \delta-\delta+\delta \gamma\right] \\
\frac{x_{1}}{x_{3}}\left[\lambda+\lambda^{2} \gamma-2 \lambda \gamma-1+\gamma+\delta+\lambda \delta \gamma-\delta \gamma\right]
\end{array}\right) \\
& \mathbf{w}^{E M}=\left(\begin{array}{c}
x_{2} \delta(1+\lambda \gamma-\gamma)(\delta+\lambda-1) \\
\frac{x_{2}}{x_{1}}\left[1+\lambda \gamma-\gamma+\lambda \delta-\delta+\delta \gamma \lambda^{2}-2 \lambda \delta \gamma+\delta \gamma\right] \\
\gamma\left(\delta \lambda^{3}-3 \delta \lambda^{2}-1+2 \delta-\delta^{2}\right) \\
\frac{x_{2}}{x_{3}}\left[2 \lambda \delta \gamma+\delta \lambda^{2}-2 \lambda \delta-2 \delta \gamma+\gamma+\delta^{2} \gamma\right]
\end{array}\right), \\
& \mathbf{w}^{E M}=\left(\begin{array}{c}
x_{3} \delta\left(\lambda \gamma+\lambda^{2}-2 \lambda-\gamma+1+\lambda \delta-\delta+\delta \gamma\right) \\
\frac{x_{3}}{x_{1}}\left[\gamma+\lambda-1+\delta \lambda^{2}-2 \lambda \delta+\delta+\lambda \delta \gamma-\delta \gamma\right] \\
\frac{x_{3}}{x_{2}}\left[2 \lambda \delta+\delta \gamma \lambda^{2}-2 \lambda \delta \gamma-2 \delta+1+\delta^{2}\right] \\
\delta \lambda^{3}-3 \delta \lambda^{2}-1+2 \delta-\delta^{2}
\end{array}\right) .
\end{aligned}
$$

Again, formulas (13)-(16) give the same principal right eigenvector, up to a scalar multiplier.

In Case $2 B(\gamma$ and $\delta$ are in different rows, and matrix size is at least $5 \times 5)$ the formulas are the following: 


$$
\begin{aligned}
& \mathbf{w}^{E M}=\left(\begin{array}{c}
\delta \lambda\left[\lambda^{3} \gamma-(n-1) \lambda^{2} \gamma-(n-3)\left(\gamma^{2}-2 \gamma+1\right)\right] \\
\frac{1}{x_{1}}\left\{\lambda^{3} \gamma-(n-2) \lambda^{2} \gamma+(n-2) \delta \gamma \lambda^{2}+[\lambda \delta+(n-4)(\delta-1)]\left(\gamma^{2}-2 \gamma+1\right)\right\} \\
\frac{1}{x_{2}} \gamma \lambda\left[\gamma+\lambda-1+\delta \lambda^{2}-2 \lambda \delta+\delta+\lambda \delta \gamma-\delta \gamma\right] \\
\frac{1}{x_{3}} \lambda\left[1+\lambda \gamma-\gamma+\lambda \delta-\delta+\delta \gamma \lambda^{2}-2 \lambda \delta \gamma+\delta \gamma\right] \\
\frac{1}{x_{4}}\left[\gamma^{2}-2 \gamma+\lambda^{2} \gamma+1+\lambda \delta-\delta \gamma \lambda^{2}-2 \lambda \delta \gamma+\lambda \gamma^{2} \delta+\lambda^{3} \delta \gamma-\delta+2 \delta \gamma-\delta \gamma^{2}\right] \\
\vdots \\
\frac{1}{x_{n-1}}\left[\gamma^{2}-2 \gamma+\lambda^{2} \gamma+1+\lambda \delta-\delta \gamma \lambda^{2}-2 \lambda \delta \gamma+\lambda \gamma^{2} \delta+\lambda^{3} \delta \gamma-\delta+2 \delta \gamma-\delta \gamma^{2}\right]
\end{array}\right) \\
& \mathbf{w}^{E M}=\left(\begin{array}{c}
x_{1}\left[\lambda^{3} \delta \gamma-(n-2) \delta \gamma \lambda^{2}-(n-4) \delta(\gamma-1)^{2}+\lambda+(n-2) \lambda^{2} \gamma-2 \lambda \gamma+\lambda \gamma^{2}+(n-4)(\gamma-1)^{2}\right] \\
\lambda\left(\lambda^{3} \gamma-(n-1) \lambda^{2} \gamma-(n-3)(\gamma-1)^{2}\right) \\
\frac{x_{1}}{x_{2}} \gamma\left(\lambda \gamma+\lambda^{2}-2 \lambda-\gamma+1+\delta \lambda-\delta+\delta \gamma\right) \\
\frac{x_{1}}{x_{3}} \lambda\left(\lambda+\lambda^{2} \gamma-2 \lambda \gamma-1+\gamma+\delta+\lambda \delta \gamma-\delta \gamma\right) \\
\frac{x_{1}}{x_{4}}\left(\lambda \gamma^{2}-2 \lambda \gamma+\lambda^{3} \gamma+\lambda-\gamma^{2}+2 \gamma-\lambda^{2} \gamma-1+\delta-2 \delta \gamma+\delta \gamma^{2}+\delta \gamma \lambda^{2}\right) \\
\vdots \\
\frac{x_{1}}{x_{n-1}}\left(\lambda \gamma^{2}-2 \lambda \gamma+\lambda^{3} \gamma+\lambda-\gamma^{2}+2 \gamma-\lambda^{2} \gamma-1+\delta-2 \delta \gamma+\delta \gamma^{2}+\delta \gamma \lambda^{2}\right)
\end{array}\right), \\
& \mathbf{w}^{E M}=\left(\begin{array}{c}
x_{2} \delta \lambda(1+\lambda \gamma-\gamma)(\delta+\lambda-1) \\
\frac{x_{2}}{x_{1}} \lambda(1+\lambda \gamma-\gamma)(1+\delta \lambda-\delta) \\
\gamma \lambda\left[\lambda^{3} \delta-(n-1) \delta \lambda^{2}-(n-3)(\delta-1)^{2}\right] \\
\frac{x_{2}}{x_{3}}\left[\delta \lambda^{3}-(n-2) \delta \lambda^{2}(1-\gamma)-2 \lambda \delta \gamma+2(n-4) \delta(1-\gamma)+\lambda \gamma+\delta^{2} \lambda \gamma+(n-4)\left(-1+\gamma-\delta^{2}+\delta^{2} \gamma\right)\right] \\
\frac{x_{2}}{x_{4}}(1+\lambda \gamma-\gamma)\left(\delta \lambda^{2}+1-2 \delta+\delta^{2}\right) \\
\vdots \\
\frac{x_{2}}{x_{n-1}}(1+\lambda \gamma-\gamma)\left(\delta \lambda^{2}+1-2 \delta+\delta^{2}\right)
\end{array}\right), \\
& \mathbf{w}^{E M}=\left(\begin{array}{c}
x_{3} \delta \lambda\left(\lambda \gamma+\lambda^{2}-2 \lambda-\gamma+1+\delta \lambda-\delta+\delta \gamma\right) \\
\frac{x_{3}}{x_{1}} \lambda(\gamma+\lambda-1)(1+\delta \lambda-\delta) \\
\frac{x_{3}}{x_{2}}\left[\lambda^{3} \delta \gamma-(n-2) \delta \lambda^{2}(\gamma-1)-2 \delta \lambda+2(n-4) \delta(\gamma-1)+\lambda+\delta^{2} \lambda+(n-4)\left(1-\gamma+\delta^{2}-\delta^{2} \gamma\right)\right] \\
\lambda\left[\delta \lambda^{3}-(n-1) \delta \lambda^{2}-(n-3)(\delta-1)^{2}\right] \\
\frac{x_{3}}{x_{4}}\left(\delta \gamma \lambda^{2}+\lambda^{3} \delta-\delta \lambda^{2}-2 \delta \lambda-2 \delta \gamma+2 \delta-1+\gamma+\lambda+\delta^{2} \lambda-\delta^{2}+\delta^{2} \gamma\right) \\
\vdots \\
\frac{x_{3}}{x_{n-1}}\left(\delta \gamma \lambda^{2}+\lambda^{3} \delta-\delta \lambda^{2}-2 \delta \lambda-2 \delta \gamma+2 \delta-1+\gamma+\lambda+\delta^{2} \lambda-\delta^{2}+\delta^{2} \gamma\right)
\end{array}\right), \\
& \mathbf{w}^{E M}=\left(\begin{array}{c}
x_{4} \delta \lambda\left(\gamma^{2}-2 \gamma+\lambda^{2} \gamma+1\right)(\delta+\lambda-1) \\
\frac{x_{4}}{x_{1}} \lambda\left(\gamma^{2}-2 \gamma+\lambda^{2} \gamma+1\right)(1+\delta \lambda-\delta) \\
\frac{x_{4}}{x_{2}} \gamma \lambda\left(\delta \gamma \lambda^{2}+\lambda^{3} \delta-\delta \lambda^{2}-2 \delta \lambda-2 \delta \gamma+2 \delta-1+\gamma+\lambda+\delta^{2} \lambda-\delta^{2}+\delta^{2} \gamma\right) \\
\frac{x_{4}}{x_{3}} \lambda\left(\delta \lambda^{2}+\lambda^{3} \delta \gamma-\delta \gamma \lambda^{2}-2 \lambda \delta \gamma-2 \delta+2 \delta \gamma-\gamma+1+\lambda \gamma+\delta^{2}+\delta^{2} \lambda \gamma-\delta^{2} \gamma\right) \\
\left(\gamma^{2}-2 \gamma+\lambda^{2} \gamma+1\right)\left(\delta \lambda^{2}+1-2 \delta+\delta^{2}\right) \\
\frac{x_{4}}{x_{5}}\left(\gamma^{2}-2 \gamma+\lambda^{2} \gamma+1\right)\left(\delta \lambda^{2}+1-2 \delta+\delta^{2}\right) \\
\vdots \\
\frac{x_{4}}{x_{n-1}}\left(\gamma^{2}-2 \gamma+\lambda^{2} \gamma+1\right)\left(\delta \lambda^{2}+1-2 \delta+\delta^{2}\right)
\end{array}\right) .
\end{aligned}
$$

Again, formulas (17)-21) give the same principal right eigenvector, up to a scalar multiplier.

Proof. The proof is similar to that of the eigenvector formulas (24)-(26) in [15]. Let us consider Case 1. Let $\mathbf{D}=\operatorname{diag}\left(1,1 / x_{1}, \ldots, 1 / x_{n-1}\right)$, and let $\mathbf{K}_{\mathbf{P}}(\lambda)=\lambda \mathbf{I}+\mathbf{U}_{1} \mathbf{V}_{1}^{T}-\mathbf{e e}^{T}$, 
with $\mathbf{U}_{1}$ and $\mathbf{V}_{1}$ as defined by (7). Since $\mathbf{D}$ is invertible, every column of the one rank matrix $\mathbf{D} \operatorname{adj}\left(\mathbf{K}_{\mathbf{P}}\left(\lambda_{\max }\right)\right) \mathbf{D}^{-1}$ is a Perron eigenvector of $\mathbf{P}$.

For Case 2, replace $\mathbf{U}_{1}$ by $\mathbf{U}_{2}$ and $\mathbf{V}_{1}$ by $\mathbf{V}_{2}$ as defined by (8).

Remark 5. Formulas (9)-21) are positive.

Proof. It is sufficient to prove the positivity of any arbitrary element of each formula, because the Perron-Frobenius theorem then guarantees the positivity for the vectors as well. The conclusions of the proofs generally follow from $x_{i}>0$ for all $i=1, \ldots, n$, $\gamma, \delta>0$ and $\lambda>n \geq 4$ (or $n \geq 5$ in Case 2B). The proof for each formula follows:

Formula (9): Positivity is apparent for $w_{1}^{E M}$.

Formula (10):

$$
\begin{aligned}
w_{1}^{E M} & =x_{1} \gamma \lambda[\delta \lambda-(n-2) \delta+\gamma+n-3] \\
& =x_{1} \gamma \lambda[\delta(\lambda-n+2)+\gamma+(n-3)]
\end{aligned}
$$

Formula (11):

$$
\begin{aligned}
w_{1}^{E M} & =x_{2} \delta \lambda[\delta+\gamma \lambda-(n-2) \gamma+n-3] \\
& =x_{2} \delta \lambda[\delta+\gamma(\lambda-n+2)+(n-3)] .
\end{aligned}
$$

Formula (12): Positivity is apparent for $w_{1}^{E M}$.

Formula (13):

$$
\begin{aligned}
w_{2}^{E M} & =\frac{1}{x_{1}}\left[\lambda^{2} \gamma-2 \lambda \gamma+\delta+2 \lambda \delta \gamma-2 \delta \gamma+\delta \gamma^{2}\right] \\
& =\frac{1}{x_{1}}\left[\lambda \gamma(\lambda-2)+\delta+2 \delta \gamma(\lambda-1)+\delta \gamma^{2}\right] .
\end{aligned}
$$

Formula (14):

$$
\begin{aligned}
w_{1}^{E M} & =x_{1}\left[\delta \gamma \lambda^{2}-2 \lambda \delta \gamma+1+2 \lambda \gamma-2 \gamma+\gamma^{2}\right] \\
& =x_{1}\left[\delta \gamma \lambda(\lambda-2)+1+2 \gamma(\lambda-1)+\gamma^{2}\right]
\end{aligned}
$$

Formula 15:

$$
\begin{aligned}
w_{1}^{E M} & =x_{2} \delta(1+\lambda \gamma-\gamma)(\delta+\lambda-1) \\
& =x_{2} \delta[1+\gamma(\lambda-1)][\delta+(\lambda-1)]
\end{aligned}
$$

Formula 16$)$ :

$$
\begin{aligned}
w_{3}^{E M} & =\frac{x_{3}}{x_{2}}\left[2 \lambda \delta+\delta \gamma \lambda^{2}-2 \lambda \delta \gamma-2 \delta+1+\delta^{2}\right] \\
& =\frac{x_{3}}{x_{2}}\left[2 \delta(\lambda-1)+\delta \gamma \lambda(\lambda-2)+1+\delta^{2}\right] .
\end{aligned}
$$

From here on in the proof, $n \geq 5$.

Formula (17): $w_{3}^{E M}$ in formula 17 is the same as $\lambda w_{3}^{E M}$ in formula 13 , which is already proven to be positive.

Formula (18): $w_{3}^{E M}$ in formula 18 is the same as $\lambda w_{3}^{E M}$ in formula (14), which is already proven to be positive. 
Formula (19):

$$
\begin{aligned}
w_{1}^{E M} & =x_{2} \delta \lambda(1+\lambda \gamma-\gamma)(\delta+\lambda-1) \\
& =x_{2} \delta \lambda[1+\gamma(\lambda-1)][\delta+(\lambda-1)]
\end{aligned}
$$

Formula 20):

$$
\begin{aligned}
w_{2}^{E M} & =\frac{x_{3}}{x_{1}} \lambda(\gamma+\lambda-1)(1+\delta \lambda-\delta) \\
& =\frac{x_{3}}{x_{1}} \lambda[\gamma+(\lambda-1)][1+\delta(\lambda-1)] .
\end{aligned}
$$

Formula 21):

$$
\begin{aligned}
w_{1}^{E M} & =x_{4} \delta \lambda\left(\gamma^{2}-2 \gamma+\lambda^{2} \gamma+1\right)(\delta+\lambda-1) \\
& =x_{4} \delta \lambda\left[\gamma^{2}+\gamma\left(\lambda^{2}-2\right)+1\right][\delta+(\lambda-1)]
\end{aligned}
$$

Using these formulas, the paper's main result can be obtained through a series of lemmas. Each of these lemmas corresponds to a directed edge in a digraph. Using these results, the direction of certain arcs can be determined. Thus, it will be shown that directed graphs of Cases 1,2A and 2B are strongly connected. By Theorem 1. efficiency of the principal right eigenvector is implied.

It follows from the positivity of $\mathbf{w}^{E M}$ (see Remark 5), that both sides of the starting inequalities of each lemma can be multiplied by the respective $w_{i}^{E M}$ without further discussion. Since there are 28 lemmas, the proofs are in the Appendix.

Cases of $\delta=1$ and $\gamma=1$ are not covered by Lemmas $1 \mathrm{a} 3 \mathrm{~h}$ due to Remark 3 .

The first group of lemmas correspond to Case 1 ( $\gamma$ and $\delta$ are in the same row), i.e., the double perturbed PCM is written in form (3).

Lemma 1a (Case 1). $\delta>1$ and $\delta \geq \gamma \Rightarrow w_{1}^{E M} / w_{2}^{E M}<\delta x_{1}$.

Proof. Using formula (10),

$$
\frac{w_{1}^{E M}}{w_{2}^{E M}}=x_{1} \frac{\gamma \lambda(\delta \lambda-(n-2) \delta+\gamma+n-3)}{\gamma \lambda^{3}-(n-1) \gamma \lambda^{2}-(n-3)\left(\gamma^{2}-2 \gamma+1\right)} .
$$

Substitute $\lambda=\lambda_{\max }$ in the characteristic polynomial $p_{\mathbf{P}}(\lambda)$ by Proposition 1 ;

$$
(-1)^{n} \lambda^{n-3}\left(\lambda^{3}-n \lambda^{2}-\left(\frac{\gamma}{\delta}+\frac{\delta}{\gamma}\right)-(n-3)\left(\gamma+\delta+\frac{1}{\gamma}+\frac{1}{\delta}\right)+4 n-10\right)=0,
$$

which can be transformed to

$$
\gamma \delta \lambda^{3}-\gamma \delta n \lambda^{2}=\gamma^{2}+\delta^{2}+(n-3)\left(\gamma^{2} \delta+\gamma \delta^{2}+\delta+\gamma\right)-\gamma \delta(4 n-10) .
$$

The statement to be proven is equivalent to

$$
\gamma \lambda(\delta \lambda+\gamma-(n-2) \delta+n-3)<\delta\left(\gamma \lambda^{3}-(n-1) \gamma \lambda^{2}-(n-3)(\gamma-1)^{2}\right) .
$$


Using (22) this is further equivalent to

$$
\left(\gamma \delta \lambda^{3}-\gamma \delta n \lambda^{2}\right)+\lambda\left(\gamma \delta(n-2)-\gamma^{2}-\gamma n+3 \gamma\right)-\delta(n-3)(\gamma-1)^{2}>0 .
$$

Now apply further equivalent transformations:

$$
\begin{gathered}
\begin{aligned}
& \gamma \lambda(\delta(n-2)-\gamma-n+3)+\gamma^{2}+\delta^{2}-(n-3)\left(\delta \gamma^{2}-2 \delta \gamma+\delta\right) \\
&+(n-3)\left(\delta^{2} \gamma+\delta \gamma^{2}+\delta+\gamma\right)-\delta \gamma(4 n-10)>0 \\
& \gamma \lambda((\delta-1)(n-3)+\delta-\gamma)+\gamma^{2}+\delta^{2} \\
&+(n-3)\left(\delta^{2} \gamma+2 \delta \gamma+\gamma\right)-4 \delta \gamma(n-3)-2 \delta \gamma>0 \\
& \gamma \lambda((\delta-1)(n-3)+(\delta-\gamma))+\gamma(n-3)(\delta-1)^{2}+(\delta-\gamma)^{2}>0 .
\end{aligned}
\end{gathered}
$$

Lemma 1b (Case 1). $\delta<1$ and $\delta \leq \gamma \Rightarrow w_{1}^{E M} / w_{2}^{E M}>\delta x_{1}$.

Proof. According to formula 10

$$
\frac{w_{1}^{E M}}{w_{2}^{E M}}=x_{1} \frac{\gamma \lambda(\delta \lambda-(n-2) \delta+\gamma+n-3)}{\gamma \lambda^{3}-(n-1) \gamma \lambda^{2}-(n-3)\left(\gamma^{2}-2 \gamma+1\right)} .
$$

Transforming (22) similar to Lemma $1 \mathrm{a}$

$$
\gamma \lambda((\delta-1)(n-3)+\delta-\gamma)+\gamma(n-3)(\delta-1)^{2}+(\delta-\gamma)^{2}<0 .
$$

Transforming this further yields

$$
\begin{gathered}
\gamma(\delta-1)(n-3)(\lambda+(\delta-1))+\gamma \lambda(\delta-\gamma)+(\delta-\gamma)^{2}<0 \\
\gamma(\delta-1)(n-3)(\lambda+(\delta-1))+(\delta-\gamma)(\gamma(\lambda-1)+\delta)<0
\end{gathered}
$$

Lemma 1c (Case 1). $\gamma>1$ and $\gamma \geq \delta \Rightarrow w_{1}^{E M} / w_{3}^{E M}<\gamma x_{2}$.

Proof. The proof follows from switching the role of $\delta$ and $\gamma$ in the proof of Lemma 1a.

Lemma 1d (Case 1). $\gamma<1$ and $\gamma \leq \delta \Rightarrow w_{1}^{E M} / w_{3}^{E M}>\gamma x_{2}$.

Proof. The proof follows from switching the role of $\delta$ and $\gamma$ in the proof of Lemma $1 \mathrm{~b}$.

Lemma 1e (Case 1). $\gamma, \delta>1 \Rightarrow w_{1}^{E M} / w_{i}^{E M}>x_{i-1}, i=4, \ldots, n$.

Proof. According to formula (9)

$$
\frac{w_{1}^{E M}}{w_{i}^{E M}}=x_{i-1} \frac{\gamma \delta \lambda(\lambda-n+1)}{\gamma+\delta+\gamma \delta \lambda-2 \gamma \delta}
$$

which means the statement to be proven is equivalent to

$$
\gamma \delta \lambda(\lambda-n+1)>\gamma+\delta+\gamma \delta \lambda-2 \gamma \delta
$$

Further equivalent transformations yield

$$
\begin{array}{r}
(\gamma \delta \lambda(\lambda-n))+(2 \gamma \delta-\gamma-\delta)>0 \\
\gamma \delta \lambda(\lambda-n)+(\delta-1)(\gamma-1)+(\delta \gamma-1)>0 .
\end{array}
$$


Lemma 1f (Case 1). $\gamma, \delta<1 \Rightarrow w_{1}^{E M} / w_{i}^{E M}<x_{i-1}, i=4, \ldots, n$.

Proof. According to formula (12)

$$
\frac{w_{1}^{E M}}{w_{i}^{E M}}=x_{i-1} \frac{\gamma \delta \lambda(\delta+\gamma+\lambda-2)}{\gamma \delta \lambda^{2}-4 \gamma \delta+\gamma+\delta+\delta^{2} \gamma+\gamma^{2} \delta} .
$$

Applying further equivalent transformations

$$
\begin{aligned}
\frac{\gamma \delta \lambda(\delta+\gamma+\lambda-2)}{\gamma \delta \lambda^{2}-4 \gamma \delta+\gamma+\delta+\delta^{2} \gamma+\gamma^{2} \delta} & <1 \\
\gamma \delta \lambda(\delta+\gamma+\lambda-2) & <\gamma \delta\left(\lambda^{2}-4\right)+\gamma+\delta+\delta^{2} \gamma+\gamma^{2} \delta \\
\lambda(\delta+\gamma+\lambda-2) & <\lambda^{2}-4+\frac{1}{\delta}+\frac{1}{\gamma}+\delta+\gamma \\
0 & <\lambda^{2}-4+\frac{1}{\delta}+\frac{1}{\gamma}+\delta+\gamma-\lambda \delta-\lambda \gamma-\lambda^{2}+2 \lambda \\
0 & <2(\lambda-2)+(1-\lambda)(\delta+\gamma)+\frac{1}{\delta}+\frac{1}{\gamma} \\
0 & <2(\lambda-1)-2+(1-\lambda)(\delta+\gamma)+\frac{1}{\delta}+\frac{1}{\gamma} \\
0 & <(\lambda-1)(2-\delta-\gamma)+\frac{1}{\delta}+\frac{1}{\gamma}-2 .
\end{aligned}
$$

Lemma 1g (Case 1). $\delta \lesseqgtr \gamma \Leftrightarrow w_{2}^{E M} / w_{3}^{E M} \gtreqless x_{2} / x_{1}$.

Proof. According to formula (9), we need to consider

$$
\frac{w_{2}^{E M}}{w_{3}^{E M}}=\frac{x_{2}}{x_{1}} \cdot \frac{\gamma \lambda-(n-2) \gamma+\delta+(n-3) \gamma \delta}{\delta \lambda-(n-2) \delta+\gamma+(n-3) \gamma \delta} \gtreqless 1 .
$$

Applying further equivalent transformations

$$
\begin{aligned}
\gamma \lambda-(n-2) \gamma+\delta+(n-3) \gamma \delta & \gtreqless \delta \lambda-(n-2) \delta+\gamma+(n-3) \gamma \delta \\
\lambda(\gamma-\delta)-(n-2)(\gamma-\delta)+\delta-\gamma & \gtreqless 0 \\
(\gamma-\delta)(\lambda-n+1) & \gtreqless 0 .
\end{aligned}
$$

The third factor is positive because $\lambda>n$.

Lemma $1 \mathbf{h}$ (Case 1$)$. $\delta \gtrless 1 \Leftrightarrow w_{2}^{E M} / w_{i}^{E M} \lessgtr x_{i-1} / x_{1}, i=4, \ldots, n$.

Proof. According to formula (9)

$$
\frac{w_{2}^{E M}}{w_{i}^{E M}}=\frac{x_{i-1}}{x_{1}} \cdot \frac{\gamma \lambda-(n-2) \gamma+\delta+(n-3) \gamma \delta}{\gamma+\delta+\gamma \delta \lambda-2 \gamma \delta} .
$$

Equivalent transformations yield

$$
\begin{aligned}
\gamma \lambda-(n-2) \gamma+\delta+(n-3) \gamma \delta & <\gamma+\delta+\gamma \delta \lambda-2 \gamma \delta \\
0 & <\gamma(\delta-1)(\lambda-n+1) .
\end{aligned}
$$

The third factor is positive because $\lambda>n$. 
Lemma 1i (Case 1). $\gamma \gtrless 1 \Leftrightarrow w_{3}^{E M} / w_{i}^{E M} \lessgtr x_{i-1} / x_{2}, i=4, \ldots, n$.

Proof. The proof follows from switching the role of $\delta$ and $\gamma$ in the proof of Lemma $1 \mathrm{~h}$.

Lemma $1 \mathbf{j}$ (Case 1). $w_{i}^{E M} / w_{j}^{E M}=x_{j-1} / x_{i-1}, i, j=4, \ldots, n$.

Proof. It follows from each of formulas (9)-12.

Corollary 1. There exists a directed cycle in each graph corresponding to Case 1 (Figure 2):

$$
\begin{aligned}
& \delta>1, \gamma>\delta: 1 \rightarrow i \rightarrow 2 \rightarrow 3 \rightarrow 1, \\
& \gamma>1, \gamma<\delta: 1 \rightarrow i \rightarrow 3 \rightarrow 2 \rightarrow 1, \\
& \delta>1, \gamma<1: 1 \rightarrow 3 \rightarrow i \rightarrow 2 \rightarrow 1, \\
& \delta<1, \gamma<\delta: 1 \rightarrow 3 \rightarrow 2 \rightarrow i \rightarrow 1, \\
& \gamma<1, \gamma>\delta: 1 \rightarrow 2 \rightarrow 3 \rightarrow i \rightarrow 1, \\
& \delta<1, \gamma>1: 1 \rightarrow 2 \rightarrow i \rightarrow 3 \rightarrow 1 .
\end{aligned}
$$

The second group of lemmas correspond to Case 2A ( $\gamma$ and $\delta$ are in different rows, and matrix size is $4 \times 4$ ), i.e., the double perturbed PCM is written in form (4).

Lemma 2a (Case $2 \mathrm{~A}) . \delta \gtrless 1 \Leftrightarrow w_{1}^{E M} / w_{2}^{E M} \lessgtr \delta x_{1}$.

Proof. Formula 16 is used for this proof. Multiplying both sides by $w_{2}^{E M}$, the statement to be proven can be written as:

$$
\begin{aligned}
x_{3} \delta\left(\lambda \gamma+\lambda^{2}-2 \lambda-\gamma+1+\right. & \lambda \delta-\delta+\delta \gamma) \\
& \lessgtr \delta x_{1} \frac{x_{3}}{x_{1}}\left(\gamma+\lambda-1+\delta \lambda^{2}-2 \lambda \delta+\delta+\lambda \delta \gamma-\delta \gamma\right) .
\end{aligned}
$$

Further equivalent transformations yield:

$$
\begin{aligned}
& 0 \lessgtr \lambda^{2} \delta-\lambda^{2}+3 \lambda-\lambda \gamma-3 \lambda \delta+\lambda \delta \gamma-2 \delta \gamma+2 \gamma+2 \delta-2 \\
& 0 \lessgtr \lambda^{2}(\delta-1)+\lambda \gamma(\delta-1)+3 \lambda(1-\delta)+2 \gamma(1-\delta)+2(\delta-1) \\
& 0 \lessgtr(\delta-1)(\lambda(\lambda-3)+\gamma(\lambda-2)+2) .
\end{aligned}
$$

The second factor on the right hand side is always positive because $\lambda>n=4$ and $\gamma, \delta>0$.

Lemma 2b (Case $2 \mathrm{~A}) . \delta>1, \gamma<1 \Rightarrow w_{1}^{E M} / w_{3}^{E M}>x_{2}$.

Proof. Formula (14) is used in this proof. Multiplying both sides by $w_{3}^{E M}$, the statement of the lemma is equivalent to:

$$
\begin{aligned}
x_{1}\left(\delta \gamma \lambda^{2}-2 \lambda \delta \gamma+1+2 \lambda \gamma-2 \gamma\right. & \left.+\gamma^{2}\right) \\
& <x_{2} \frac{x_{1}}{x_{2}} \gamma\left(\lambda \gamma+\lambda^{2}-2 \lambda-\gamma+1+\lambda \delta-\delta+\delta \gamma\right) .
\end{aligned}
$$

Further equivalent transformations yield:

$$
0<\lambda^{2} \gamma-\lambda^{2} \gamma \delta-4 \lambda \gamma+\lambda \gamma^{2}+3 \lambda \delta \gamma-2 \gamma^{2}+3 \gamma+\delta \gamma^{2}-\delta \gamma-1
$$




$$
\begin{aligned}
& 0<\lambda^{2} \gamma(1-\delta)+\lambda \gamma(\gamma-1)+3 \lambda \gamma(\delta-1)+(\gamma-1)+2 \gamma(1-\gamma)+\delta \gamma(\gamma-1) \\
& 0<(1-\delta) \lambda \gamma(\lambda-3)+(\gamma-1)(\gamma(\lambda-2)+\delta \gamma+1)
\end{aligned}
$$

The second factor on the right hand side is always positive because $\lambda>n=4$ and $\gamma, \delta>0$.

Lemma 2c (Case 2A). $\delta<1, \gamma>1 \Rightarrow w_{1}^{E M} / w_{3}^{E M}<x_{2}$.

Proof. The proof follows from the right hand side of (24) being positive in the case of $\delta<1, \gamma>1$.

Lemma 2d (Case 2A). $\delta, \gamma<1 \Rightarrow w_{1}^{E M} / w_{4}^{E M}<x_{3}$.

Proof. Again, formula (14) is used for this proof. Multiplying both sides by $w_{4}^{E M}$, the statement to be proven is equivalent to:

$$
\begin{aligned}
x_{1}\left(\delta \gamma \lambda^{2}-2 \lambda \delta \gamma+1+2 \lambda \gamma-2 \gamma\right. & \left.+\gamma^{2}\right) \\
& <x_{3} \frac{x_{1}}{x_{3}}\left(\lambda+\lambda^{2} \gamma-2 \lambda \gamma-1+\gamma+\delta+\lambda \delta \gamma-\delta \gamma\right) .
\end{aligned}
$$

Further equivalent transformations yield:

$$
\begin{array}{r}
\lambda^{2} \gamma \delta-\lambda^{2} \gamma+4 \lambda \gamma-3 \lambda \delta \gamma-\lambda+\gamma^{2}-3 \gamma+\delta \gamma-\delta+2<0 \\
(\delta-1)\left(\lambda^{2} \gamma-3 \lambda \gamma\right)+(\gamma-1)(\lambda+\gamma-2+\delta)<0 \\
(\delta-1) \lambda \gamma(\lambda-3)+(\gamma-1)((\lambda-2)+\gamma+\delta)<0
\end{array}
$$

The left hand side is negative if $\gamma, \delta<1$, because $\lambda>n=4$.

Lemma 2e (Case 2A). $\delta, \gamma>1 \Rightarrow w_{1}^{E M} / w_{4}^{E M}>x_{3}$.

Proof. The proof follows from the left hand side of (25) being positive if $\gamma, \delta>1$.

Lemma 2f (Case 2A). $\delta, \gamma<1 \Rightarrow w_{2}^{E M} / w_{3}^{E M}>x_{2} / x_{1}$.

Proof. Formula 13 is used in this proof. Multiplying both sides by $w_{3}^{E M}$, the statement of the lemma can be written as:

$$
\begin{aligned}
\frac{1}{x_{1}}\left(\lambda^{2} \gamma-2 \lambda \gamma+\delta+2 \lambda \delta \gamma-2 \delta \gamma\right. & \left.+\delta \gamma^{2}\right) \\
& >\frac{x_{2}}{x_{1}} \frac{1}{x_{2}} \gamma\left(\gamma+\lambda-1+\delta \lambda^{2}-2 \lambda \delta+\delta+\lambda \delta \gamma-\delta \gamma\right) .
\end{aligned}
$$

Further equivalent transformations yield:

$$
\begin{aligned}
& 0>\lambda^{2} \gamma \delta-\lambda^{2} \gamma-4 \lambda \delta \gamma+3 \lambda \gamma+\lambda \delta \gamma^{2}+\gamma^{2}-2 \delta \gamma^{2}+3 \delta \gamma-\gamma-\delta \\
& 0>(\delta-1)\left(\lambda^{2} \gamma-3 \lambda \gamma\right)+(\gamma-1)(\lambda \delta \gamma-2 \delta \gamma+\delta+\gamma) \\
& 0>(\delta-1) \lambda \gamma(\lambda-3)+(\gamma-1)(\delta \gamma(\lambda-2)+\delta+\gamma) .
\end{aligned}
$$

The right hand side is negative if $\delta, \gamma<1$, because $\lambda>n=4$.

Lemma 2g (Case 2A). $\delta, \gamma>1 \Rightarrow w_{2}^{E M} / w_{3}^{E M}<x_{2} / x_{1}$. 
Proof. The proof follows from the right hand side of (26) being positive if $\delta, \gamma>1$.

Lemma 2h (Case 2A). $\delta<1, \gamma>1 \Rightarrow w_{2}^{E M} / w_{4}^{E M}>x_{3} / x_{1}$.

Proof. Again, formula 13 is used in this proof. Multiplying both sides by $w_{4}^{E M}$, the statement to be proven is equivalent to:

$$
\begin{aligned}
\frac{1}{x_{1}}\left(\lambda^{2} \gamma-2 \lambda \gamma+\delta+2 \lambda \delta \gamma-\right. & \left.2 \delta \gamma+\delta \gamma^{2}\right) \\
& >\frac{x_{3}}{x_{1}} \frac{1}{x_{3}}\left(1+\lambda \gamma-\gamma+\lambda \delta-\delta+\delta \gamma \lambda^{2}-2 \lambda \delta \gamma+\delta \gamma\right) .
\end{aligned}
$$

Further equivalent transformations yield:

$$
\begin{aligned}
& 0>\lambda^{2} \gamma \delta-\lambda^{2} \gamma-4 \lambda \delta \gamma+3 \lambda \gamma+\lambda \delta+3 \delta \gamma-\delta \gamma^{2}-2 \delta-\gamma+1 \\
& 0>(\delta-1)\left(\lambda^{2} \gamma-3 \lambda \gamma\right)+(1-\gamma)(\lambda \delta-2 \delta+\delta \gamma+1) \\
& 0>(\delta-1) \lambda \gamma(\lambda-3)+(1-\gamma)(\delta(\lambda-2)+\delta \gamma+1)
\end{aligned}
$$

The right hand side of (27) is negative, if $\delta<1, \gamma>1$, because $\lambda>n=4$.

Lemma 2i (Case 2A). $\delta>1, \gamma<1 \Rightarrow w_{2}^{E M} / w_{4}^{E M}<x_{3} / x_{1}$.

Proof. The proof follows from the right hand side of (27) being positive if $\delta>1, \gamma<$ 1.

Lemma 2 j (Case $2 \mathrm{~A}) . \gamma \gtrless 1 \Leftrightarrow w_{3}^{E M} / w_{4}^{E M} \lessgtr \gamma x_{3} / x_{2}$.

Proof. Once again, formula 13 is used for the proof. Multiplying both sides by $w_{4}^{E M}$, the first statement (for $\gamma>1$ ) becomes equivalent to:

$$
\begin{aligned}
\frac{1}{x_{2}} \gamma\left(\gamma+\lambda-1+\delta \lambda^{2}-\right. & 2 \lambda \delta+\delta+\lambda \delta \gamma-\delta \gamma) \\
& \lessgtr \gamma \frac{x_{3}}{x_{2}} \frac{1}{x_{3}}\left(1+\lambda \gamma-\gamma+\lambda \delta-\delta+\delta \gamma \lambda^{2}-2 \lambda \delta \gamma+\delta \gamma\right) .
\end{aligned}
$$

Applying further equivalent transformations:

$$
\begin{aligned}
& 0 \lessgtr \lambda^{2} \delta \gamma-\lambda^{2} \delta-3 \lambda \delta \gamma+3 \lambda \delta+\lambda \gamma-\lambda+2 \delta \gamma-2 \delta-2 \gamma+2 \\
& 0 \lessgtr(\gamma-1)\left(\lambda^{2} \delta-3 \lambda \delta+\lambda+2 \delta-2\right) \\
& 0 \lessgtr(\gamma-1)(\lambda \delta(\lambda-3)+(\lambda-2)+2 \delta) .
\end{aligned}
$$

The second factor on the right hand side of $(29)$ is positive because $\lambda>n=4$ and $\gamma, \delta>0$.

Corollary 2. There exists a directed cycle in each graph corresponding to Case 2A (Figure 3):

$$
\begin{aligned}
& \delta>1, \gamma>1: 1 \rightarrow 4 \rightarrow 3 \rightarrow 2 \rightarrow 1, \\
& \delta>1, \gamma<1: 1 \rightarrow 3 \rightarrow 4 \rightarrow 2 \rightarrow 1, \\
& \delta<1, \gamma<1: 1 \rightarrow 2 \rightarrow 3 \rightarrow 4 \rightarrow 1, \\
& \delta<1, \gamma>1: 1 \rightarrow 2 \rightarrow 4 \rightarrow 3 \rightarrow 1 .
\end{aligned}
$$


The last group of lemmas correspond to Case $2 \mathrm{~B}$, when $\gamma$ and $\delta$ are in different rows, and matrix size is at least $5 \times 5$, i.e., the double perturbed PCM is written in form (5).

Lemma 3a (Case 2B). $\gamma \gtrless 1 \Leftrightarrow w_{3}^{E M} / w_{4}^{E M} \lessgtr \gamma x_{3} / x_{2}$.

Proof. Using formula (17) the proof is similar to the proof of Lemma 2j, the only difference is in (28) where both sides are multiplied by $\lambda$, which immediately cancel each other.

Lemma 3b (Case 2B). $\delta \gtrless 1 \Leftrightarrow w_{1}^{E M} / w_{2}^{E M} \lessgtr \delta x_{1}$.

Proof. Using formula (21) the proof is similar to the proof of Lemma 2a, the only difference is in (23) where both sides (the formula for $w_{1}^{E M}$ and $w_{2}^{E M}$ ) are multiplied by $\lambda$, which immediately cancel each other. This may not be apparent about $w_{2}^{E M}$, but

$$
(\gamma+\lambda-1)(1+\delta \lambda-\delta)=\gamma+\lambda-1+\lambda \gamma \delta+\lambda^{2} \delta-\lambda \delta-\gamma \delta-\lambda \delta+\delta
$$

which, after reduction, gives the same formula.

Lemma 3c (Case 2B). $\delta \gtrless 1 \Leftrightarrow w_{1}^{E M} / w_{i}^{E M} \gtrless x_{i-1}, i=5, \ldots, n$.

Proof. Formula 19 is used for this proof.

$$
\begin{aligned}
x_{2} \delta \lambda(1+\lambda \gamma-\gamma)(\delta+\lambda-1) & \gtrless x_{i-1} \frac{x_{2}}{x_{i-1}}(1+\lambda \gamma-\gamma)\left(\delta \lambda^{2}+1-2 \delta+\delta^{2}\right) \\
\lambda \delta^{2}+\lambda^{2} \delta-\lambda \delta & \gtrless \delta \lambda^{2}+1-2 \delta+\delta^{2} \\
\lambda \delta(\delta-1)+\delta(1-\delta)+(\delta-1) & \gtrless 0 \\
(\delta-1)(\delta(\lambda-1)+1) & \gtrless 0 .
\end{aligned}
$$

The second factor on the left hand side is always positive because $\lambda>n \geq 5$ and $\delta>0$.

Lemma 3d (Case 2B). $\delta \gtrless 1 \Leftrightarrow w_{2}^{E M} / w_{i}^{E M} \lessgtr x_{i-1} / x_{1}, i=5, \ldots, n$.

Proof. Again, formula (19) is used in the proof.

$$
\begin{aligned}
\frac{x_{2}}{x_{1}} \lambda(1+\lambda \gamma-\gamma)(1+\delta \lambda-\delta) & \lessgtr \frac{x_{i-1}}{x_{1}} \frac{x_{2}}{x_{i-1}}(1+\lambda \gamma-\gamma)\left(\delta \lambda^{2}+1-2 \delta+\delta^{2}\right) \\
\lambda+\lambda^{2} \delta-\delta \lambda & \lessgtr \delta \lambda^{2}+1-2 \delta+\delta^{2} \\
0 & \lessgtr \lambda \delta-\lambda+\delta^{2}-2 \delta+1 \\
0 & \lessgtr \lambda(\delta-1)+(\delta-1)^{2} \\
& \lessgtr \lessgtr(\delta-1)((\lambda-1)+\delta) .
\end{aligned}
$$

The second factor on the right hand side is always positive because $\lambda>n \geq 5$ and $\delta>0$.

Lemma 3e (Case $2 \mathrm{~B}) \cdot \gamma \gtrless 1 \Leftrightarrow w_{3}^{E M} / w_{i}^{E M} \gtrless x_{i-1} / x_{2}, i=5, \ldots, n$. 
Proof. Formula 18 is used in this proof.

$$
\begin{aligned}
& \frac{x_{1}}{x_{2}} \gamma \lambda\left(\lambda \gamma+\lambda^{2}-2 \lambda-\gamma+1+\delta \lambda-\delta+\delta \gamma\right) \\
& \quad \gtrless \frac{x_{4}}{x_{2}} \frac{x_{1}}{x_{4}}\left(\lambda \gamma^{2}-2 \lambda \gamma+\lambda^{3} \gamma+\lambda-\gamma^{2}+2 \gamma-\lambda^{2} \gamma-1+\delta-2 \delta \gamma+\delta \gamma^{2}+\delta \gamma \lambda^{2}\right) .
\end{aligned}
$$

Further equivalent transformations yield:

$$
\begin{aligned}
\lambda^{2} \gamma^{2}-\lambda^{2} \gamma-2 \lambda \gamma^{2}+3 \lambda \gamma+\lambda \gamma^{2} \delta-\lambda \delta \gamma-\lambda+\gamma^{2}-2 \gamma+1-\delta+2 \delta \gamma-\delta \gamma^{2} & \gtrless 0 \\
(\gamma-1)\left(\lambda^{2} \gamma-2 \lambda \gamma+\lambda+\lambda \delta \gamma+(\gamma-1)-\delta(\gamma-1)\right) & \gtrless 0 \\
(\gamma-1)(\lambda \gamma(\lambda-2)+(\lambda-1)+\delta \gamma(\lambda-1)+\gamma+\delta) & \gtrless 0 .
\end{aligned}
$$

The second factor on the left hand side is always positive because $\lambda>n \geq 5$ and $\gamma, \delta>0$.

\section{Lemma 3f.}

$\gamma>1, \delta<1 \Rightarrow w_{2}^{E M} / w_{4}^{E M}>x_{3} / x_{1}$.

$\gamma<1, \delta>1 \Rightarrow w_{2}^{E M} / w_{4}^{E M}<x_{3} / x_{1}$.

Proof. Instead of the statement of the lemma, we will prove the following stronger statement:

$$
\gamma \gtreqless \delta \Leftrightarrow w_{2}^{E M} / w_{4}^{E M} \gtreqless x_{3} / x_{1} .
$$

Formula $(21)$ is used in this proof.

$$
\begin{aligned}
& \frac{x_{4}}{x_{1}} \lambda\left(\gamma^{2}-2 \gamma+\lambda^{2} \gamma+1\right)(1+\delta \lambda-\delta) \\
& \gtreqless \frac{x_{3}}{x_{1}} \frac{x_{4}}{x_{3}} \lambda\left(\delta \lambda^{2}+\lambda^{3} \delta \gamma-\delta \gamma \lambda^{2}-2 \lambda \delta \gamma-2 \delta+2 \delta \gamma-\gamma+1+\lambda \gamma+\delta^{2}+\delta^{2} \lambda \gamma-\delta^{2} \gamma\right) .
\end{aligned}
$$

This is further equivalent to

$$
\begin{aligned}
\gamma^{2}+\lambda \gamma^{2} \delta-\gamma^{2} \delta-2 \gamma-2 \lambda \gamma \delta+2 \gamma \delta+\lambda^{2} \gamma+\lambda^{3} \gamma \delta-\lambda^{2} \gamma \delta+1+\lambda \delta-\delta \\
\\
\equiv \lambda^{2} \delta+\lambda^{3} \gamma \delta-\lambda^{2} \gamma \delta-2 \lambda \gamma \delta-2 \delta+2 \gamma \delta-\gamma+1+\lambda \gamma+\delta^{2}+\lambda \gamma \delta^{2}-\gamma \delta^{2} .
\end{aligned}
$$

Further equivalent transformations yield

$$
\begin{gathered}
\lambda^{2} \gamma-\lambda^{2} \delta+\lambda \delta-\lambda \gamma+\lambda \gamma^{2} \delta-\lambda \gamma \delta^{2}+\gamma^{2}-\delta^{2}+\gamma \delta^{2}-\gamma^{2} \delta+2 \delta-2 \gamma+\gamma-\delta \gtreqless 0 \\
\lambda^{2}(\gamma-\delta)+\lambda(\delta-\gamma)+\lambda \gamma \delta(\gamma-\delta)+(\gamma+\delta)(\gamma-\delta)+\gamma \delta(\delta-\gamma)+2(\delta-\gamma)+(\gamma-\delta) \gtreqless 0 \\
(\gamma-\delta)\left(\lambda^{2}-\lambda+\lambda \gamma \delta+\gamma+\delta-\gamma \delta-1\right) \gtreqless 0 \\
(\gamma-\delta)\left(\lambda^{2}-2 \lambda+\lambda \gamma \delta-\gamma \delta+\lambda-1+\gamma+\delta\right) \gtreqless 0 \\
(\gamma-\delta)(\lambda(\lambda-2)+\gamma \delta(\lambda-1)+(\lambda-1)+\gamma+\delta) \gtreqless 0 .
\end{gathered}
$$

The second factor on the left hand side is always positive because $\lambda>n \geq 5$ and $\gamma, \delta>0$ 
Lemma 3g (Case 2B).

$\gamma, \delta>1 \Rightarrow w_{1}^{E M} / w_{4}^{E M}>x_{3}$.

$\gamma, \delta<1 \Rightarrow w_{1}^{E M} / w_{4}^{E M}<x_{3}$.

Proof. Instead of the above statement, we will prove the following stronger statement:

$$
\gamma \delta \gtreqless 1 \Leftrightarrow w_{1}^{E M} / w_{4}^{E M} \gtreqless x_{3} .
$$

Formula (21) is used in this proof.

$$
\begin{aligned}
& x_{4} \delta \lambda\left(\gamma^{2}-2 \gamma+\lambda^{2} \gamma+1\right)(\delta+\lambda-1) \\
& \gtreqless x_{3} \frac{x_{4}}{x_{3}} \lambda\left(\delta \lambda^{2}+\lambda^{3} \delta \gamma-\delta \gamma \lambda^{2}-2 \lambda \delta \gamma-2 \delta+2 \delta \gamma-\gamma+1+\lambda \gamma+\delta^{2}+\delta^{2} \lambda \gamma-\delta^{2} \gamma\right) .
\end{aligned}
$$

Further equivalent transformations yield:

$$
\begin{array}{r}
\lambda^{2} \delta^{2} \gamma-\lambda^{2} \delta+\lambda \gamma^{2} \delta-\lambda \gamma \delta^{2}+\lambda \delta-\lambda \gamma+\gamma^{2} \delta^{2}-\gamma \delta^{2}-\delta \gamma^{2}+\delta+\gamma-1 \gtreqless 0 \\
(\delta \gamma-1)\left(\lambda^{2} \delta+\lambda \gamma-\lambda \delta+\delta \gamma+1-\delta-\gamma\right) \gtreqless 0 \\
(\delta \gamma-1)(\lambda \delta(\lambda-2)+\gamma(\lambda-1)+\delta(\lambda-1)+\delta \gamma+1) \gtreqless 0 .
\end{array}
$$

The second factor is always positive because $\lambda>n \geq 5$ and $\gamma, \delta>0$. The first factor is positive exactly if $\gamma \delta>1$, and negative exactly if $\gamma \delta<1$.

Lemma 3h (Case 2B). $w_{i}^{E M} / w_{j}^{E M}=x_{j-1} / x_{i-1}, i, j=5, \ldots, n$.

Proof. It follows from each of formulas 17 - 21).

Corollary 3. There exists a directed cycle in each graph corresponding to Case 2B (Figure 4):

$$
\begin{aligned}
& \delta>1, \gamma>1: 1 \rightarrow 4 \rightarrow 3 \rightarrow i \rightarrow 2 \rightarrow 1 \\
& \delta>1, \gamma<1: 1 \rightarrow i \rightarrow 3 \rightarrow 4 \rightarrow 2 \rightarrow 1, \\
& \delta<1, \gamma<1: 1 \rightarrow 2 \rightarrow i \rightarrow 3 \rightarrow 4 \rightarrow 1 \\
& \delta<1, \gamma>1: 1 \rightarrow 2 \rightarrow 4 \rightarrow 3 \rightarrow i \rightarrow 1
\end{aligned}
$$

